\title{
"L'IMPRONTA ESTERNA DEL NOSTRO IO". NOTE INTORNO AI PRIMI LINEAMENTI DEL DIRITTO SULLA PROPRIA IMMAGINE
}

\author{
"THE EXTERNAL IMPRINT OF OUR SELF". NOTES ON THE FIRST \\ FEATURES OF THE RIGHT TO ONE'S OWN IMAGE
}

\author{
Elisabetta Fusar Poli \\ Università degli studi di Brescia
}

\begin{abstract}
English: The paper addresses the "very modern" right to one's own image, in the unfolding of its dynamics through the kaleidoscopic and crucial Nineteenth-Twentieth century turning. Attention is paid in particular to legal scholarship and case law, which have helped to outline and then define an autonomous subjective right having as its object one's personal image, and to find for it a systematization within the legal order, from the end of the Nineteenth century to publication of the First Book of the Civil Code. Technical innovation in progress raises the issue, as the reproduction and wide spread of the human physiognomy, facilitated by the potentialities of modern devices and means of communication, sharpens the urgency of protection for what appears to be both strictly inherent to the person and related to the social projection of the individual. From the defense of honor and reputation, to protection from undue intrusions into the personal sphere, the complex issue is at the crossroads of different legal areas and opens in the Twentieth century to unexpected developments and transformations. In fact, it gradually fits into a more complex conception of the person, which emerges from a scientific reflection transversal to the fields of sociology, anthropology, philosophy, biology, and turns in the direction of 'personal identity'.
\end{abstract}

Keywords: Right to One's Own Image; Civil Codification; Rights related to Personality; Personal Identity Right; Copyright; European Legal History; Contemporary History

Abstract Italiano: Lo scritto affronta il «modernissimo» diritto sulla propria immagine, nello svolgersi delle sue dinamiche attraverso il caleidoscopico e cruciale tornante otto-novecentesco. L'attenzione è rivolta agli apporti dottrinali e giurisprudenziali che hanno contribuito a tratteggiare e poi definire un diritto soggettivo autonomo avente ad oggetto la propria immagine personale, e a trovare per esso una collocazione all'interno dell'ordine giuridico, dalla fine dell'Ottocento alla pubblicazione del Libro primo del Codice Civile. II tema è sollecitato dall'innovazione tecnica in corso nel periodo considerato e dagli stimoli di una società ormai in fase protomediatica, nella quale la riproduzione della fisionomia umana, agevolata dalle potenzialità dei moderni mezzi di comunicazione, acuisce l'urgenza di una tutela per ciò che appare strettamente inerente alla persona e, al contempo, proiezione sociale dell'individuo, interfaccia fra la sua dimensione privata e quella pubblica. Dalla tutela dell'onore e della reputazione, alla protezione dalle incursio-

* Italian Review of Legal History, 7 (2021), n. 11, pagg. 377-417

* https://riviste.unimi.it/index.php/irlh/index

* ISSN 2464-8914 - DOI 10.54103/2464-8914/16893. Articolo pubblicato sotto Licenza CC-BY. 
ni indebite nella sfera personale, il complesso tema è al crocevia fra diversi spazi giuridici e si apre nel Novecento a inattesi sviluppi e trasformazioni. Si inserisce, infatti, gradualmente, entro una più complessa concezione di persona, che emerge da una riflessione scientifica trasversale ai campi della sociologia, antropologia, filosofia, biologia, e volge in direzione della 'identità personale'.

Parole chiave: Diritto sulla propria immagine; Codificazione civile; Diritti della personalità; Diritto all'identità personale; Diritto d'autore; Storia del diritto europeo; Storia contemporanea

Sommario: 1. Fra intimità e socialità. - 2. A partire dal Codice: privato e pubblico, individuale e collettivo, nella dimensione personale e del diritto. - 3. "Un incontro di teoria e pratica». - 3.1. Intrecci normativi e intuizioni. - 3.2. "Una giurisprudenza circospetta, pari all'altezza dei nuovi rapporti di fatto». - 3.3. Fardelli d'onore. - 3.4. Lunghe costruzioni. 3.4.1. Giusnaturalismi e "tedescherie". - 3.4.2. Suggestioni novecentesche fra ragione e principi. - 4. Direzioni.

"Man mag von rechts kommen oder von links - man wird sich daran gewöhnen müssen, darauf angesehen zu werden, woher man kommt. Man wird es, seinerseits, den andern anzusehen haben ${ }^{1}$.

W. Benjamin, Kleine Geschichte der Photographie

\section{Fra intimità e socialità}

Le sistemazioni offerte dalla trattatistica di diritto civile degli ultimi decenni sovente indugiano sulla complessa affermazione precodicistica del cosiddetto 'diritto all'immagine', species ormai accolta nel problematico genus dei diritti della personalità ${ }^{2}$, suggerendo l'opportunità di indagarne la mutevole fisionomia anche da un angolo visuale prettamente storico-giuridico. In tale differente prospettiva, più che scovare a ritroso nel passato remote ascendenze ${ }^{3}$, mi pare possa essere

\footnotetext{
${ }^{1}$ La citazione dell'opera, edita originariamente nel 1931, è tratta da Benjamin, 1991, p. 381.

2 La categoria dei 'diritti della personalità' è "fortemente problematica» ancora nell'attualità, per le stesse ragioni per le quali lo è nel torno di tempo qui considerato: un sostanziale scollamento fra "le strutture concettuali che ne orientano la comprensione» e la realtà, che è «in continua e rapida evoluzione» (Alpa, Resta, 2019, p. 162), oggi come negli anni a cavaliere fra XIX e XX secolo. Del resto, «il diritto della personalità [...] è in sé un concetto 'storico', nel senso che il suo significato varia con il variare della cultura e della civiltà giuridica che lo esprime» e del modello antropologico, esso stesso variabile nel tempo, che funge da riferimento (ivi, p. 164).

${ }^{3}$ In tema di 'diritto alla immagine' (o 'sulla immagine', com'è preferito nei primi decenni del Novecento) non mancano tentativi relativamente recenti di offrire ricostruzioni storiche (cfr. in particolare Cionti, 1998 e Cionti, 2000) che, pur dando ampiamente conto della polifonia degli apporti rilevanti del dibattito otto-novecentesco sul tema, sovrappongono le voci, a discapito di una valorizzazione del processo graduale d'emersione,
} 
di particolare interesse scomporre in vettori e forze la vicenda di tale peculiare e "modernissimo» diritto, nello svolgersi delle sue dinamiche - anzitutto entro la scienza giuridica e la giurisprudenza - attraverso il caleidoscopico e cruciale tornante otto-novecentesco. Esito atteso della scomposizione che mi accingo a effettuare sarà, dunque, non tanto la ricostruzione di un dettagliato affresco diacronico, ma piuttosto qualcosa di simile all'ingresso in uno speciale laboratorio, nel mentre delle attività che vi si compiono.

Si tratta delle attività che hanno portato a tratteggiare e poi definire un diritto soggettivo autonomo avente ad oggetto la propria immagine personale, oggetto di rappresentazione, riproduzione e diffusione, e a trovare per esso una collocazione all'interno dell'ordine giuridico, dal secondo Ottocento all'approvazione del Libro primo del Codice nel dicembre del 1938.

È un percorso non certo lineare, quello qui proposto, dunque particolarmente stimolante, almeno da due profili. Da un lato, esso investe vari ambiti del diritto, sollecitati dall'innovazione tecnica, dagli stimoli di una società ormai in fase protomediatica ${ }^{4}$, abbacinata e al contempo intimorita dai nuovi e sofisticati strumenti di riproduzione della fisionomia umana, e dalle potenzialità dei moderni mezzi di comunicazione. In effetti, dagli ultimi decenni dell'Ottocento, è palpabile la crescente e concreta sensibilità per una tutela dell'immagine di sé, catturata da strumenti tecnici sempre più accurati e invadenti (l'apparecchio per la fotografia istantanea ${ }^{5}$ materializza l'idea della riproducibilità negli informati commenti a sentenza e nei saggi d'epoca ${ }^{6}$ ) che incrementano la possibilità di diffusione dell'effigie personale e pertanto della sua conoscibilità e riconoscibilità, oltreché

definizione e sistemazione del diritto medesimo. Per rigore d'approccio e accuratezza dei richiami alle fonti, cito, fra i contributi giusprivatistici che affrontano il diritto sull'immagine collocandolo entro il quadro storico dei diritti della personalità, Messinetti, 1983; De Vita, 1988; Busnelli, 1995; Zeno-Zencovich, 1995; Dogliotti, 1999; Resta, 2007; Canestrari, Ferrando, Mazzoni, Rodotà, Zatti, 2011; Alpa, Resta, 2019.

${ }^{4}$ Ferrara, 1942, p. 7.

${ }^{5}$ «Fotografi di professione e dilettanti si incontrano ovunque, tanto nei luoghi pubblici che nelle case private, sempre pronti a fissare sulle lastre delle loro macchine le sembianze delle persone, consenzienti o no, e spesso l'indiscrezione dei kodak si spinge fino al punto da non rispettare neppure i segreti della vita privata» (Von Blume, 1903, c. 723).

${ }^{6}$ Saggi, articoli, note e sentenze soprattutto dei primi decenni del Novecento appalesano una chiara consapevolezza della portata dirompente dell'apparecchio fotografico, particolarmente a sviluppo istantaneo, nonché della tecnica cinematografica: preoccupazioni sulle possibilità di intrusione nella sfera privata fronteggiano i crescenti interessi anche di natura patrimoniale suscitati dalle possibilità di diffusione dell'immagine. Spunti di particolare interesse sul tema della fotografia nel contesto del rapporto fra innovazione tecnica e diritto, fra XIX e XX secolo, sono in Martello, 2018, pp. 53 ss.. Fra le fonti coeve all'arco temporale preso in considerazione, rimando soprattutto a Bucciante, 1911; Ferrara, 1937 e, per la riflessione d'area francofona, patria delle prime innovazioni in campo fotografico, Bigeon, 1894. 
del suo improprio utilizzo.

$L^{\prime}$ immagine quale «impronta esterna del nostro io» ${ }^{7}$ è qualcosa di strettamente inerente alla persona e al contempo è esposta ai terzi, è proiezione sociale dell'individuo, interfaccia fra la sua dimensione privata e quella pubblica. Per questo, sul confine mobile fra le due dimensioni, si staglia dapprima l'esigenza di tutela da eventuali lesioni dell'onore e della reputazione, perpetrate per il tramite di immagini verisimili e dettagliate, poi più ampiamente l'esigenza di protezione dalle incursioni indebite nella sfera personale ( "la curiosità non può elevarsi a dignità $d^{\prime}$ interesse sociale» $\left.!^{8}\right)$. Ci addentreremo a breve lungo questi vettori d'indagine, che intersecano e connettono diversi spazi giuridici, sia che si parli di ambiti disciplinari, non sempre nitidamente delineati, sia che ci si riferisca alla dimensione dottrinale e giurisprudenziale, quest'ultima particolarmente fertile e foriera di soluzioni atte a colmare lacune e vaghezze normative.

Al rilievo circa la trasversalità della "elegante questione», com'è in più occasioni definita sulle pagine delle riviste giuridiche, si aggiunge poi un ulteriore profilo di particolare interesse, che è alimentato dalla dimensione sovranazionale investita dal tema. Quella dell'immagine, della sua riproduzione e diffusione, è infatti una questione che svela anche inedite prospettive alla scienza giuridica e alla giurisprudenza nazionale, entrambe spronate a spingersi oltre i confini del quadro normativo italiano, ad aprirsi alla ricerca di spunti e riferimenti (da assimilare, o da cui prendere debite distanze) nell'ambito dalla coeva esperienza giuridica internazionale, soprattutto francese e tedesca. Un campo privilegiato in cui, dunque, è messo alla prova quel policromo Italian style otto-novecentesco che la storiografia ha efficacemente evidenziato ${ }^{9}$.

Sono solo pochi e sintetici spunti introduttivi, questi, che possono ora lasciare il campo al cuore della vicenda giuridica proposta: mi appresto a proiettarla su uno schermo d'eccezione (il Codice civile), con l'intento di individuare più nitidamente i suoi protagonisti, le trame e gli snodi.

\section{A partire dal Codice: privato e pubblico, individuale e collettivo, nella dimensione personale e del diritto}

La complessità di questioni dogmatiche e sistematiche, teoriche e pratiche, suscitate dal tema della 'immagine propria' e persistenti ancora fra anni '30 e '40 del Novecento è tracciabile fra le righe dei lavori intorno al Codice civile, dal Progetto preliminare alla definitiva versione del suo testo, con attenzione al Titolo primo del Libro primo, ove la «persona fisica» è protagonista.

Non mi attarderò in una minuziosa ricostruzione di tali vicende, testimoniate dalla scienza giuridica che la ha vissute nel loro dispiegarsi, e già lumeggiate,

\footnotetext{
${ }^{7}$ Degni, 1939, p. 201.

${ }^{8}$ Ibidem.

${ }^{9}$ Lacché, 2015.
} 
talora con pregevole profondità di campo storico, anche da recente civilistica ${ }^{10}$. Piuttosto, la messa a fuoco sarà qui diretta sul processo che porta al faticoso ingresso della tutela dell'immagine propria nella elaborazione del futuro articolo 10 del Codice. I motivi di tale (accidentato) percorso si ricollegano direttamente all'emersione di quella che, non senza contrasti dottrinali e incertezze (peraltro destinate a perdurare anche decenni oltre la codificazione), si sta affermando quale autonoma categoria di diritti soggettivi inerenti alla persona: i 'diritti della personalità'.

Le cautele del legislatore sono implicite nel testo codicistico, sin dalla sua prima versione, e possono essere ricostruite assemblando alcuni significativi passaggi delle relazioni e dei verbali che conservano traccia sintetica, ma eloquente, dei momenti più intensi del dibattito istituzionale sin dalla elaborazione del Progetto preliminare.

Già dal dato letterale si evincono alcuni elementi significativi: anzitutto la liaison strettissima fra nome e immagine (accomunati nella rubrica e nel testo dell'art. 11, sull' «Abuso del nome o dell'immagine altrui») ${ }^{11}$, che va diluendosi nei vari passaggi dell'iter normativo, attraverso i quali l'immagine si affranca dal 'diritto al nome', guadagnando un articolo autonomo che regola, però, il solo profilo rimediale e non riconosce espressamente un autonomo diritto soggettivo.

${ }^{10}$ Alpa, Resta, 2019, pp. 239 ss., in particolare pp. 269-270; Busnelli, 1995, pp. 113-116. Risalendo alle prime ricostruzioni, si vedano De Sanctis, 1937; Degni, 1939, pp. 202 ss.; Ferrara, 1942, pp. 17-40. II richiamo alle fonti concerne in particolare: Commissione Reale per la riforma dei codici, 1931, Codice Civile, Primo libro. Progetto e Relazione, Istituto poligrafico dello Stato, Roma; Ministero di Grazia e Giustizia, 1933, Lavori preparatori per la riforma del Codice civile; Osservazioni e proposte sul progetto del Libro Primo, vol.: I: Disposizioni preliminari - Titolo I: delle persone fisiche - Titolo II: Delle persone giuridiche, Tip. delle Mantellate, Roma, pp. 364-376; Commissione Reale per la riforma dei codici, 1936, Codice civile. Libro primo. Progetto definitivo e relazione del Guardasigilli on. Solmi, Istituto poligrafico dello Stato, Roma; Commissione delle Assemblee legislative per i codici civile, di procedura civile, di commercio e per la marina mercantile, 1937, Atti della Commissione parlamentare chiamata a dare il proprio parere sul progetto del Libro Primo del Codice civile "Delle persone" (art. 2 della Legge 30 dicembre 1923-II, n. 2814 e art. 2 e 3 della Legge 24 dicembre 1925-IV, n. 2260), Roma, Tip. del Senato, pp. 364 ss.; Ministero di Grazia e Giustizia, 1943, Relazione alla Maestà del Re Imperatore del Ministro Guardasigilli (Grandi) presentata all'udienza del 16 marzo 1942-XX per l'approvazione del testo del 'Codice Civile, in Codice Civile. Testo e Relazione ministeriale, Istituto Poligrafico dello Stato, Roma.

${ }^{11}$ Art. 11 (Abuso del nome o dell'immagine altrui): "Qualora il nome di una persona sia stato usato in una pubblicazione in modo da arrecare pregiudizio al suo decoro o alla sua reputazione, l'autorità giudiziaria, su richiesta dell'interessato, può disporre che cessi l'abuso, salva sempre l'azione per il risarcimento dei danni. La stessa tutela compete alla persona la cui immagine e quella dei genitori, del coniuge, dei figli in età minore siano state esposte o pubblicate con pregiudizio suo o dei detti congiunti. Il giudice terrà conto se fu osservato $[\ldots]$ ». 
II nucleo normativo originario, che attraverso i vari passaggi legislativi sopravvive sino alla pubblicazione del Libro primo, limita la disciplina codicistica alla previsione di strumenti di tutela anzitutto per il caso in cui sia causato un pregiudizio al decoro o alla reputazione della persona effigiata (o dei suoi congiunti) attraverso la pubblicazione dell'immagine. La versione definitiva trasfusa nel testo dell'art. 10 amplia poi la fattispecie rubricata come abuso, estendendola anche all'ipotesi in cui l'esposizione o pubblicazione avvenga "fuori dai casi previsti dalla legge». Il che altro non è, in quel preciso momento storico, se non un implicito richiamo alle disposizioni della legge per la protezione del diritto d'autore, in tema di «Diritti relativi al ritratto» ovvero all'art. 11 del Regio decreto legge 7 novembre 1925 n. 1950, rimpiazzato di lì a breve della nuova legge 22 aprile 1941, n. 633 ${ }^{12}$. Le reazioni 'a caldo' sulle norme sono testimonianza di un certo scetticismo ${ }^{13} \mathrm{e}$, al contempo, di una ancora latente percezione delle potenzialità d'espansione in via interpretativa dell'articolo 10 , con i suoi impliciti o trasversali corollari.

Ai nostri fini, tali reazioni sono alcune delle tracce disseminate nelle fonti, utili per cogliere gli apporti alla progressiva elaborazione teorico-pratica del diritto riconosciuto al soggetto sulla propria immagine. E la ricerca delle tracce ci invita ad addentrarci, in prima battuta, nelle pieghe del processo codificatorio, da cui emergono chiaramente i nodi teorico-sistematici, ma anche di politica legislativa ai quali possono essere imputate alcune cautele, suscitate dal dubbio circa l'effettiva sussistenza di un diritto soggettivo e sulla sua conseguente collocazione nell'ordinamento giuridico. Alle soglie della codificazione, infatti, ancora non pare esservi unanime, né assodata convergenza di posizioni in merito a problemi di non poco conto da un profilo giuridico, a partire dalla configurabilità di un'autonoma categoria dei 'diritti della personalità' entro la quale pare ascrivibile 'd'ufficio' quello all'immagine.

In avvio dei lavori, i differenti approcci al tema sono svelati già nei riscontri al Progetto preliminare. La magistratura, da un lato, aderendo alla tendenza interpretativa attestatasi nella giurisprudenza degli ultimi tre decenni precedenti il Codice (e vi torneremo a breve), è nel senso di un riconoscimento generale di una tutela della personalità entro la quale ricondurre la tutela dell'immagine senza specifica esigenza di costruire intorno ad essa un diritto autonomo ${ }^{14}$. II Consiglio

\footnotetext{
${ }^{12} \mathrm{Cfr}$. infra, 3.1. Si noti che lo stesso Ministro guardasigilli, a giustificazione della soppressione, nel progetto definitivo sottoposto alla Commissione parlamentare, di qualsivoglia disposizione in tema di diritto all'immagine, evidenzia l'inopportunità (e di qui, la superfluità) della previsione: Progetto definitivo e relazione, p. 14.

${ }^{13}$ Poco efficace se non persino pleonastico è immediatamente giudicato da alcune voci l'esito dei faticosi lavori. Si nota, ad esempio, che «il sistema adottato non è stato felice e, indubbiamente, l'interpretazione dell'art. 10 darà luogo a molte incertezze. Viene spontanea la domanda: con quella norma si riconosce o non un diritto assoluto all'immagine?» (Degni, 1939, p. 203).

${ }^{14} \mathrm{Si}$ vedano i riscontri della corte d'Appello di Catanzaro e Venezia, in particolare, di cui alle Osservazioni e proposte, pp. 365-366.
} 
di Stato, dal canto suo, ligio a un'impostazione conservatrice e di fatto scarsamente coinvolto nella conflittualità originata dalla questione, evidenzia che un «diritto sulla propria immagine non è stato riconosciuto», ma piuttosto il legislatore intende "reprimere una lesione al diritto all'onore e decoro di una persona ${ }^{15}$. Le associazioni rappresentative di avvocati e procuratori, apprezzando la questione soprattutto dal lato delle tutele esperibili, si concentrano sulla inadeguatezza della disposizione, per via degli strumenti rimediali, che sono originariamente previsti solo in caso di pregiudizio di onore e reputazione ${ }^{16}$. Insomma, che le varie quaestiones sottese al laconico testo preliminare siano disputate è palese.

Una sottovalutazione di queste e ulteriori critiche e delle esigenze registrate soprattutto nella casistica sottoposta sempre più frequentemente alla cognizione delle corti nazionali, unita a una fiducia nella esaustività e rapida approvazione delle nuove disposizioni di riforma del diritto d'autore, alla quale si lavora già da tempo pressoché in parallelo al Codice ${ }^{17}$, induce così a espungere radicalmente dal successivo Progetto ministeriale ogni disposizione in tema di immagine. Una scelta forse pilatesca, che immediatamente suscita reazione nella Commissione parlamentare chiamata a dare il suo parere sul progetto del Libro primo: il deputato e avvocato livornese Edoardo Rotigliano, escludendo che «il diritto all'immagine possa essere considerato alla stregua del diritto di autore», rivendica perentoriamente che esso debba piuttosto tornare ad essere trattato «nella sede delle persone fisiche» ${ }^{18}$.

È risoluta la contrapposizione del senatore e magistrato Eduardo Piola Caselli, autorevole artefice novecentesco del diritto d'autore italiano, nonché convinto sostenitore della svolta collettivista anche nel contesto del Codice ${ }^{19}$. II presiden-

\section{Ibidem.}

${ }^{16}$ Ivi, pp. 371-376. Ma vi è anche chi riconduce la questione al gius punitivo piuttosto che al diritto privato (Sindacato Avvocati e Procuratori di Salerno), o chi si sporge oltre le critiche prevalenti al testo, come la Commissione Reale Avvocati di Trieste e Capodistria (ivi, pp. 375-376), per la quale "gli interessi morali di una persona possono essere offesi col mezzo di una pubblicazione anche in altro modo che non sia l'uso del suo nome o della sua immagine. Non deve essere consentito che i fatti della vita privata di una persona e di quella dei suoi famigliari vengano tratti alla luce col mezzo della pubblicità. Esiste, come suol dirsi, un diritto alla persona non solo sulla sua immagine fisica, ma anche sulla sua immagine spirituale». E i riferimenti, nell'affermare questa versione abbozzata di diritto alla riservatezza, sono alla giurisprudenza austriaca, attraverso la quale si evidenzia il rischio di interpretazioni troppo ardite e forzate, che la disposizione esaminata susciterebbe per la sua intrinseca insufficienza, ove si volesse raggiungere un risultato di tutela «desiderato e desiderabile» per fattispecie analoghe concretamente pregiudizievoli.

${ }^{17}$ Moscati, 2020, pp. 208-213.

${ }^{18}$ Atti della Commissione parlamentare, pp. 58-59.

${ }^{19}$ Su Eduardo Piola Caselli (1868-1943), brillante magistrato e uomo delle istituzioni, nazionali e internazionali, mi limito a richiamare Fusar Poli, 2012, pp. 232 ss. e passim, con i riferimenti alle fonti ivi menzionate. Autorevole 'tecnico' del diritto, con una specifica competenza in campi del diritto sollecitati dalla innovazione tecnologica e dallo sviluppo 
te D'Amelio compone il dissidio fra i due e propone l'inserimento di un nuovo e autonomo articolo per l'immagine, ripristinando per esso uno spazio ad hoc: è il nuovo articolo 9-bis, che pare voler assecondare l'idea di un ampliamento della tutela, oltre l'ipotesi di lesione all'onore o reputazione ${ }^{20}$.

Ma alla seduta del primo dicembre 1936 si ripropone il confronto fra Rotigliano e Piola Caselli, che segna punti a favore dell'uno e dell'altro. La proposta avanzata in via principale da Piola Caselli è infatti una cancellazione dell'articolo riproposto, sulla scorta di una considerazione assorbente: il diritto sulla immagine propria è un portato dei reflussi giusnaturalistici veicolati da recenti teorizzazioni tedesche sui diritti della personalità, che ha trovato nella dottrina italiana, e pure nella Commissione reale promotrice della versione originaria del testo, un favore maggiore che nello stesso contesto germanico. II "concetto pericolosissimo» di un diritto assoluto sull'immagine propria, conseguenza di una riproposta «concezione giusnaturalistica del diritto privato" di stampo individualistico, denegata dal fascismo ${ }^{21}$, si assomma all'evidenza che "la protezione dei diritti generali della persona appartenga al diritto pubblico ${ }^{22}$ e non debba dunque essere inclusa

dei mezzi di comunicazione, e altresì profondo conoscitore della dottrina tedesca e francese, è autore di numerosi scritti che approfondiscono i principali istituti della proprietà intellettuale e del diritto industriale lungo la prima metà del Novecento (cfr. anche Moscati, 2020, pp. 212 ss.).

${ }^{20}$ Art. 9-bis: "Qualora l'immagine di una persona o dei genitori, del coniuge o dei figli di età minore sia stata esposta o pubblicata con pregiudizio suo e dei suoi detti congiunti, l'autorità giudiziaria, su richiesta dell'interessato, può disporre che cessi l'abuso, salva sempre l'azione per il risarcimento dei danni».

${ }^{21}$ Sull'eccesso individualistico, il magistrato si è peraltro già speso all'epoca delle sue prime note a sentenza in tema, peraltro organicamente riversate nella prima edizione del suo Trattato, avviato nel 1905 e pubblicato in versione definitiva nel 1907 (Piola Caselli, 1907). Più ampiamente sull'individualismo, anche in connessione con la codificazione, cfr. Alvazzi del Frate, $2020^{2}$, nonché, in tema della crisi della radice individualista del modello costituzionale italiano in età fascista cfr. Sciumè, 2012, pp. 248 ss..

${ }^{22}$ Commissione Parlamentare, verbale seduta del $1^{\circ}$ dicembre 1936, pp. 60-63 e relazione sui lavori, alle pp. 737-738. Dalle parole di Piola Caselli, che echeggiano il pensiero di Jellinek e i suoi diritti pubblici soggettivi (Caravale, 2016, pp. 62 ss.), emergono alcuni temi cari alla politica legislativa fascista, che affiorano dalle testimonianze scritte di quella "opera intellettuale» (per usare le parole di Mariano d'Amelio) che è la riforma dei codici. È interessante notare che, nel riassumere la vocazione complessiva del Titolo primo del Libro primo, Fulvio Maroi (1891-1954), dalle pagine del Commentario diretto da Mariano d'Amelio, rimarca la piena compatibilità fra affermazione della persona umana, centralità dello Stato e corporativismo. Il complesso di norme destinato alle persone fisiche collocato in apertura al Codice non è, dunque, una mera trovata sistematica per blandire la dottrina maggioritaria, ma è "premessa indispensabile in un codice che vuole riflettere i principî direttivi della dottrina del Fascismo il quale, lungi da degradare e mortificare la persona umana, intende tutelarla nella sua integrità (fisica, razziale e morale), potenziarla nella sua capacità produttiva, proteggerla nella sua dignità e nei suoi essenziali attribu- 
nella codificazione civile.

Le pressioni in chiave sistematica di Rotigliano prevalgono: «nel titolo del Codice dove si parla delle persone fisiche» ha "sede naturale» il diritto all'immagine 'propria', per quanto Piola Caselli si sforzi, con convinta adesione del professore Pietro Cogliolo, di restringere il perimetro della codificazione civile ai soli diritti patrimoniali e familiari. Trova alfine decisiva fortuna la proposta avanzata «in via subordinatissima» da Piola Caselli, il quale, ricordando pragmaticamente le «esigenze della vita sociale moderna», che impongono una tolleranza crescente alla diffusione dell'immagine, e lamentando il rischio che, anche in via interpretativa, possa configurarsi un diritto soggettivo assoluto, nel caso fosse previsto rimedio per qualunque pregiudizio arrecato dalla diffusione dell'immagine, ottiene l'approvazione della versione cristallizzata nel testo definitivo del Libro primo.

Ridimensionata e completata ${ }^{23}$, la fattispecie d'abuso dell'immagine per il quale possa disporsi l'intervento dell'attività giudiziaria e il risarcimento, è configurata nel Codice qualora

l'immagine di una persona o dei genitori, del coniuge o dei figli sia stata esposta o pubblicata fuori dei casi in cui l'esposizione o la pubblicazione è dalla legge consentita, ovvero con pregiudizio al decoro o alla reputazione della persona stessa o dei detti congiunti $[\ldots]^{24}$

L'esplicazione del legislatore è immediata: all'atto della pubblicazione del Libro primo è precisato che la «norma del nuovo codice integra quella dell'art. 11 della vigente legge sui diritti di autore R.D.L. 7 novembre 1925, n. $1050 »^{25}$.

Intercorsa successivamente l'approvazione della nuova legge speciale, il diritto all'immagine appare, al momento dell'entrata in vigore del Codice nel suo complesso,

già disciplinato dalla legge 22 aprile 1941, n. 633, sulla protezione del diritto di autore (articoli 96 e 97), ma non poteva nella parte del codice civile, relativa ai diritti della personalità, mancare una norma che affermasse il diritto sul proprio ritratto ${ }^{26}$.

Con l'esito concreto, possiamo aggiungere, che effettuando un ipotetico bilanciamento, «il diritto all'immagine prevale sul diritto d'autore di colui che ha effettuato il ritratto $»^{27}$.

ti» (Maroi, 1940, p. 82). Sul rapporto fra codificazione e fascismo, anche successivamente alla caduta del regime, sono sempre assai stimolanti le riflessioni di Cappellini, 1999.

${ }^{23}$ La proposta della Commissione, che si è sostanziata nell'aggiunta dell'articolo 9-bis, contempla infatti l'avvertenza finale «che con questa disposizione non s'intende toccare quanto è regolato dalla legge sui diritti d'autore»; il caveat si converte nell'inciso del definitivo articolo 10: «fuori dei casi in cui l'esposizione è dalla legge consentita».

${ }^{24}$ Art. 10: Abuso dell'immagine altrui.

${ }^{25}$ Maroi, 1940, p. 104.

${ }^{26}$ Relazione alla Maestà del Re, § 40, p. 20.

${ }^{27}$ De Cupis, 1959, p. 263. 


\section{3. "Un incontro di teoria e pratica»}

I lavori alla codificazione autorizzano a una considerazione d'avvio: l'attività giurisprudenziale ${ }^{28}$, prima, e le elaborazioni teorico-sistematiche della dottrina, poi, precedono e nutrono, lungo i primi quattro decenni del Novecento, le scelte normative. In realtà, si registra anche qualche precoce manifestazione di sensibilità e attenzione nella scienza giuridica nazionale, sulla scorta della circolazione di soluzioni giurisprudenziali, soprattutto transalpine ${ }^{29}$, e di costruzioni teoriche specialmente tedesche, già nel corso del secondo Ottocento. Fonti dalle quali emerge a poco a poco un droit à l'image o un Recht am eigenen Bilde, captato dai sensibili ricettori dei giuristi nazionali più aperti alle sollecitazioni da oltreconfine.

Indagando gli spazi di interazione fra giurisprudenza e dottrina, italiana ed europea, sulla scorta delle tracce problematiche che abbiamo raccolto attraverso le cronache istituzionali nel precedente paragrafo, assumiamo ora di poter procedere, prendendo le mosse dal rapporto fra i diritti d'autore e il diritto sull'immagine propria, un rapporto normativamente impossibile in Italia sino al 1925, e che dunque deve essere esplorato ricercando connessioni che prescindano dal dato positivo nazionale. A ben guardare, si tratta di connessioni e inneschi anche pregiuridici, di apparente banalità, ma di tutta rilevanza anzitutto agli occhi del giurista di fine Ottocento e inizio Novecento: è stato necessario "arrivare alla fotografia e alla diffusione di immagini fedeli - precisa Luigi Ferrara ${ }^{30}$ in uno dei suoi acuti approfondimenti in tema - per poter avere un largo sviluppo delle

\footnotetext{
${ }^{28} \mathrm{Nel}$ nostro caso, si può dire che «la pratica, senza soppiantare la teoria, le porge il suo alimento vitale» (De Cupis, 1959, p. 5, anche per la citazione che intitola il paragrafo).

${ }^{29}$ Che siano soprattutto le decisioni francesi di metà Ottocento l'incubatrice delle «più decise e decisive» affermazioni in tema di diritto sulla propria immagine, è evidenziato in Amar, 1874, pp. 369 ss. (ove l'Autore cita giurisprudenza tratta dal repertorio Dalloz e dagli Annales de la propriété industrielle), nonché, anche per la giurisprudenza estera del Novecento e ancor più vigorosamente, in Ferrara, 1903, cc. 281 ss. e Ricca-Barberis, 1958.

${ }^{30}$ A Luigi Ferrara (1875-1950), avvocato e professore di diritto privato anche a Roma (Zarro, 2015), dobbiamo alcuni fra i più acuti e sensibili contributi al tema del diritto sulla propria immagine, nei decenni che precedono la codificazione, sin dall'avvio del Novecento. Il suo sguardo perspicace precorre temi della riflessione giuridica contemporanea, che connettono il diritto all'innovazione tecnica e alla creatività artistica. II suo II diritto sulla propria immagine nel nuovo Codice civile e nella nuova legge sul diritto d'autore, edito a Roma nel 1942, rappresenta la chiosa ideale della sua intensa attività scientifica in tema. Cogliendo un momento determinante della storia giuridica, il trattato esalta l'intreccio, evidente anche nella rispettiva elaborazione, fra i due fondamentali testi normativi di riferimento, ovvero il Codice civile e la legge sul diritto d'autore. Premessa una breve ricostruzione storica (pp. 7-15), Ferrara si addentra nelle legislazioni straniere, per poi concentrarsi sulla giurisprudenza e sulla dottrina internazionale e nazionale. La seconda parte del trattato considera questioni specifiche più problematiche, emerse nei decenni, e le ultime tendenze in tema di «riserbo e intimità della vita privata» (pp. 352-354).
} 
controversie sui ritratti e più genericamente sulla riproduzione delle proprie sembianze» ${ }^{31}$.

Il passaggio dal ritratto a pennello, o scolpito nel marmo, alla fotografia (persino istantanea!) complica la prospettiva meramente contrattuale fra artista e committente, regolata nelle prime rudimentali norme europee ottocentesche ${ }^{32}$, aggiungendo alle dinamiche di diritto un nuovo protagonista, ovvero il soggetto ritrattato.

\subsection{Intrecci normativi e intuizioni}

Lo sguardo teso oltre i confini nazionali è tendenzialmente diffuso nella scienza giuridica italiana a cavaliere fra '800 e '900 e, in modo ancor più evidente, connota gli specialisti dei nuovi ambiti del diritto legati all'innovazione tecnica e alla nascente economia industriale. Fra loro, è l'avvocato e libero docente torinese Moise $\mathrm{Amar}^{33}$, che per primo si addentra nelle problematiche complesse sollevate dalla diffusione dell'immagine della persona umana, nel suo trattato Dei diritti degli autori di opere dell'ingegno, edito nel 1874 , vero e proprio manuale teorico-pratico dall'appeal internazionale.

Amar è un pioniere: avvia l'insegnamento del diritto industriale (all'epoca inclusivo, da un profilo disciplinare, anche della materia dei diritti d'autore) presso l'ateneo della capitale sabauda, sin dal 1877, ed è avvocato di fama, specializzato soprattutto nel diritto commerciale e delle nuove questioni della proprietà intellettuale. Il vivo interesse, pratico e al contempo teorico, per «quei rapporti di diritto che, per esser frutto di condizioni prettamente moderne, non trovavano nella dottrina tradizionale adeguata considerazione ${ }^{34}$ è la sua cifra, che si unisce a una profonda conoscenza, anche diretta, per ragioni professionali, non solo della dottrina tedesca ma anche del diritto d'Oltralpe. Da lì (e soprattutto dalla giurisprudenza francese) attingendo i suoi principali riferimenti, egli mette a fuoco alcune efficaci soluzioni interpretative che tentano un contemperamento fra

\footnotetext{
${ }^{31}$ Ferrara, 1903, c. 281.

32 Piola Caselli, 1903, p. 634 e Ferrara, 1942, pp. 17-34. Con particolare riferimento alla disciplina tedesca che, ancorandosi soprattutto alla riflessione dottrinale, offre prima riferimenti fuorvianti (ovvero una precoce ed "errata» disciplina di legge, che attribuisce al committente e non al ritrattato o all'autore del ritratto i diritti di riproduzione e disposizione sull'immagine: Ferrara, 1903, cc. 296-301; Dusi, 1906, p. 228), e poi, dal 1907 (col Gesetz betreffend das Urheberrecht an Werken der bildenden Künste und der Photographie, specialmente $\S 22$ ) validi spunti anche al legislatore nazionale (cfr. Alpa, Resta, 2019, pp. 180-191).

${ }^{33}$ Sulla carriera professionale e accademica, per quanto sempre da libero docente, di Moisè Amar (1844-1914), si veda Fusar Poli, 2018. La figura di Amar potrà essere ulteriormente illuminata grazie allo spoglio delle cospicue carte, personali e professionali, versate dai discendenti all'Archivio Ebraico Terracini.

${ }^{34}$ Ivi, p. 173.
} 
istanze individualistiche ed esigenze sociali ${ }^{35}$ in tema di immagine e diritti su di essa.

II nesso oggettivo fra autore del ritratto ed effigiato alimenta una fisiologica ascrizione della questione di una possibile tutela della immagine personale entro gli spazi giuridici - essi stessi problematici e in corso di definizione - del diritto d'autore, ma con la precisazione che «debbansi ben distinguere i diritti d'autore dagli altri diritti che le leggi di tutti i popoli civili tutelano più o meno esplicitamente», ivi incluso il diritto «insito nella natura umana [...] della piena e libera disponibilità della propria persona». Considerato che su di sé la persona «ha un dominio pieno, e così l'ha pure sulla propria immagine", si deve concludere che «questa è padrona assoluta di permetterlo o non che si faccia il suo ritratto [...] e che, dato tale permesso, essa può ritirarlo» ${ }^{36}$.

Ciò stabilito, questa forma di "dominio assoluto» sull'immagine propria ${ }^{37}$ è perfettamente conciliabile col diritto dell'autore della sua riproduzione: il diritto all'immagine non si risolve dunque sic et simpliciter nel diritto d'autore, ma deve dialogare con esso, in forma di contrappunto, ovvero quale limite alla piena espansione dei diritti e facoltà dell'autore. II dialogo, tuttavia, non è ancora cristallizzato in norma e la materia del diritto d'autore è, al momento delle intuizioni di Amar, affidata a una legge, assai esile, del 1865, destinata ad essere superficialmente rimaneggiata in un testo unico nel $1882^{38}$. La lacunosità delle norme lascia spazi ampi di discussione e interpretazione, entro i quali si affacciano temi destinati a intersecarsi con le esigenze crescenti e concrete di tutela dell'immagine.

In quegli anni e anche successivamente, nel Novecento, si discute della natura patrimoniale e al contempo morale (strettamente pertinente alla persona) ${ }^{39}$ dei diritti e delle facoltà che si riconducono all'autore e, da un profilo teorico-dogma-

\footnotetext{
${ }^{35}$ Prendo in prestito, parafrasandole, le parole di De Cupis, che negli anni Cinquanta mette a fuoco il conflitto fra le due contrapposte sfere d'interessi e istanze, quella «individualistica, secondo la quale la persona dev'essere arbitra delle proprie fattezze» e quella, di pari valore, che riunisce le esigenze sociali di conoscenza e critica della persona (De Cupis, 1959, p. 260).

${ }^{36}$ Amar, 1874, pp. 366-367.

${ }^{37}$ Ma si veda la critica al ricorso alla categoria del 'dominio' di Amar, espressa in Rosmini, 1890; critica ripresa, ma non condivisa, da Ferrara, 1903, cc. 303-304.

${ }^{38}$ La precedente legge 25 giugno 1865, n. 2337 e il successivo Decreto 19 settembre 1882, n. 1012 non contengono specifiche previsioni.

${ }^{39} \mathrm{Se}$, infatti, dobbiamo a Eduardo Piola Caselli il merito di aver veicolato efficacemente (grazie a una robusta struttura teorica arricchita anche da apporti dottrinali stranieri) nella normativa nazionale i diritti morali d'autore, la «apertura ai diritti morali» risale già al secondo Ottocento: ne sono protagonisti italiani Luigi Borsari, Enrico Rosmini, Moisè Amar, Antonio Turchiarulo, Ferruccio Foà. In merito si vedano le accurate pagine di Moscati, 2020, pp. 204 ss. (p. 195 per richiami alla teoria 'dualista' di Josef Kohler), anche per i preziosi riferimenti bibliografici e alle fonti, nonché Moscati, 2021 con stimolanti riflessioni in prospettiva europea.
} 
tico, si oscilla fra il pilastro proprietario, che rimanda alla 'proprietà incorporale' di ascendenza francese ${ }^{40}$, e le provocazioni dagli Immaterialgüterrechts e Persönlichkeitsrechts di matrice tedesca. Sono pungoli teorici ampiamente accolti anche in Italia, che ancor più innervano e rinsaldano, anche concettualmente, i nessi fra tutela dell'immagine e diritti d'autore attraverso la riflessione intorno ai diritti della personalità.

Dal profilo dell'oggetto di tutela normativa, peraltro, si aggiunge la questione specifica dell'immagine fotografica, che stenta a trovare spazio nel contesto del diritto d'autore "perché il lavoro che produce la fotografia è principalmente fisico-chimico ${ }^{41}$. Ampia è la casistica in tema, in assenza di disposizioni ad hoc ${ }^{42}$; il dubbio è sciolto a livello nazionale ed internazionale nel senso di una assimilazione delle opere fotografiche alle altre coperte dal diritto d'autore (sebbene generalmente con una tutela più ristretta), ciò configurando un'ulteriore tappa di avvicinamento anche concettuale fra tutela dei diritti d'autore e tutela dell'immagine.

Dal recepimento degli esiti giurisprudenziali e dottrinali dei decenni precedenti, nonché dal riutilizzo del materiale elaborato nel corso dei conati normativi che sono andati succedendosi in Italia sin dal $1902^{43}$, con l'autorevole paternità di Piola Caselli è approvato il Regio decreto legge 7 novembre 1925 n. 1950 che, accolta fra le numerose innovazioni la tutela autonoma del diritto morale d'auto-

${ }^{40}$ Cfr. sul punto Moscati, 2020, pp. 91 ss. e la bibliografia, ivi richiamata.

${ }^{41}$ Valerio, 1930, p. 43.

${ }^{42}$ Valerio, 1930, pp. 42 ss. e 315 ss.; Piola Caselli, 1927, pp. 196-199 e Piola Caselli, 1943, pp. 484-493 e 500-509; Ferrara, 1901, p. 30 ss. Per un esame degli orientamenti prevalenti in dottrina cfr. Martello, pp. 57 ss. Fra la giurisprudenza più interessante, rappresentativa anche delle difficoltà di qualificazione da parte della Suprema Corte, si veda la vicenda della ditta Naya, finita due volte in Cassazione a Roma, una nel 1891 e una nel 1892, per fotografie di incisioni: al di là delle pretese delle parti e delle questioni di diritto internazionale connesse al tema delle annessioni delle provincie venete al Regno, si dibatte se, ai sensi della disciplina vigente in materia di diritto d'autore, tali fotografie siano traduzioni o riproduzioni meccaniche (sentenze 12 marzo 1891 e Foro Italiano, 16 gennaio 1892 riportate rispettivamente in Foro Italiano, 1891, 16, cc. 197-206 e 1892, 17, cc. 153-162, nonché, per sviluppi giurisprudenziali in tema, Cass. Roma, Costanzo e Martinazzi ricc., 8 novembre 1904, in Foro Italiano, 1905, 30, cc, 194 ss., con ampio commento in nota di Piola Caselli).

${ }^{43}$ Alimentato in particolare dal lavoro svolto dalla commissione ad hoc presieduta da Vittorio Polacco fra 1917 e 1919. Sulle difficoltà della Commissione Polacco, istituita «con l'incarico di esaminare, studiare e proporre le modificazioni da introdurre alla vigente legge sui diritti spettanti agli autori delle opere dell'ingegno", si veda in particolare Moscati, 2020, pp. 208 ss.. Per i dettagli sul laborioso percorso normativo che sin da principio ha contemplato l'esigenza di regolare in qualche modo la riproduzione dell'immagine, sull'esempio di altre nazioni europee e in particolare della Germania, si veda Ferrara, 1942, pp. 34-40 (le pagine precedenti dell'Autore offrono un sintetico spaccato del quadro normativo estero in tema). 
re (all'articolo 16), ospita anche la prima fisionomia normativa che il legislatore italiano conferisce alla tutela dell'immagine propria.

L'articolo 11 la contiene, prevedendo che:

Il ritratto di una persona non può essere pubblicato o messo in commercio senza il consenso espresso o tacito della persona medesima e, dopo la sua morte, del coniuge e dei figli, o, in loro mancanza dei genitori, e, mancando il coniuge, i figli e i genitori, degli altri ascendenti e discendenti diretti. La persona che ha dato il consenso può revocarlo, salvo l'obbligo del risarcimento dei danni. E' libera la pubblicazione del ritratto quando abbia scopi scientifici, didattici, e, in genere, culturali, o si riferisca a fatti o avvenimenti di interesse pubblico o svoltisi in pubblico ${ }^{44}$.

Il dettato normativo, che ruota intorno al consenso, è un primo punto d'arrivo, un precipitato storico delle tappe compiute dall'interprete e delle acquisizioni sempre più salde guadagnate per gradi dalla scienza giuridica. Amar ne ha in un certo senso tracciato le coordinate, pur in mancanza di appigli normativi nazionali, attingendo a piene mani dalla giurisprudenza francese e dalla personale prospettiva teorica, non digiuna di letture tedesche: il ruolo chiave del consenso, revocabile, e l'esigenza di discriminare il contesto della riproduzione dell'immagine, in funzione della rilevanza pubblica dello stesso, ora trovano accoglienza espressa nella norma.

Nell'immediatezza della entrata in vigore del decreto n. 1950, già si ragiona però di una nuova riforma, alla quale si dedica, pressoché in continuità con i lavori della precedente, sempre Eduardo Piola Caselli ${ }^{45}$, che abbiamo visto prota-

${ }^{44}$ II Decreto, recante Disposizioni sul diritto di autore, pubblicato nella G.U. 20 novembre 1925 n. 270, è convertito dalla legge 18 marzo 1926, n. 562 e abrogato dalla legge 22 aprile 1941, n. 633. Per una ricostruzione ricca di riferimenti alle fonti, si veda l'imprescindibile Moscati, 2020, pp. 212 ss., nonché, a caldo, Valerio, 1926 e Piola Caselli, 1927. Ne emerge il principale passaggio dogmatico registrato nel corso dei lavori, dal 1917 al 1925, per mano di Piola Caselli: dall'impostazione 'dualista', alla 'monista', ovvero ai diritti d'autore come «diritti di natura giuridica mista, personale e patrimoniale, con una configurazione autonoma dei diritti patrimoniali» (Moscati, 2020, p. 213).

${ }^{45}$ Ferrara, 1942; Piola Caselli, 1943, pp. 5 ss.: sollecitata dalle novità introdotte dalla Convenzione di Roma del 1928 (cfr. Moscati, 2015), già nel 1936 è costituita la prima commissione di studio ai fini della riforma normativa (Piola Caselli, 1943, pp. 8-9). In particolare, nel Codice del diritto di autore, il commentario edito nel 1943 per iniziativa e opera del magistrato e principale artefice dei due testi di legge novecenteschi in materia di diritti d'autore, appare chiaro sin dai lavori, riportati nelle prime centosettanta pagine del testo, che i tre articoli in tema di ritratto non suscitano particolari esigenze di chiarimento, evidentemente appalesandosi quale consolidazione dei principi e regole elaborati dalla giurisprudenza e dottrina. Solo l'articolo 98 è oggetto di esplicazione in quanto disposizione nuova rispetto alla legge del 1925: la norma in questione si propone di elidere le incertezze in tema di sfruttamento economico da parte del committente, generate negli anni soprattutto sulla scorta della legislazione germanica ed anglosassone, sancendo di fatto i diritti di sfruttamento patrimoniale dell'opera in capo al fotografo, salvo patto con- 
gonista anche degli accesi dibattiti intorno al Libro primo del Codice. Egli impersona una sorta di prezioso link intellettuale fra le due discipline, quella generale codicistica e quella speciale, entrambe in corso di elaborazione, un ponte che si stabilisce anche nel punto di intersezione normativa rappresentato dal definitivo articolo 10 del Codice.

La novità degli articoli 96 e 98 della Legge n. 633 del 22 aprile $1941^{46}$ non è tanto in ciò che dispongono, che in larga misura riproduce, dettagliandolo, il contenuto del vecchio articolo 11 del 1925, quanto nella circostanza che essi partecipano ormai del valore normativo generale del novello Codice. Si stabilisce così una sorta di incorporazione organica degli uni nell'altro, per tramite del richiamo implicito che i lavori preparatori ci permettono inequivocabilmente di identificare, riflesso della sedimentazione degli approdi dottrinali e giurisprudenziali.

\section{2. "Una giurisprudenza circospetta, pari all'altezza dei nuovi rapporti di fatto»}

Le sentenze che in qualche misura toccano il tema della tutela dell'immagine, emesse negli anni precedenti il cruciale articolo 11 della legge sul diritto d'autore del 1925 e riportate nelle principali effemeridi giuridiche, sono relativamente poche, ma destano curiosità e interesse scientifico. Si intuisce presto la portata critica del tema, che risveglia e scuote costruzioni dogmatiche un po' intorpidite e che, coagulando apporti teorici trasversali, diventa gradualmente fecondo bacino interpretativo. Nella casistica "si delinea ed attua la nuova entità giuridica» del diritto sulla propria immagine, al punto che il legislatore potrà limitarsi, come abbiamo visto, a «formulare ciò che si è elaborato rebus ipsis dictantibus et ne-

trario (ivi, p. 509).

${ }^{46}$ Per immediato riscontro testuale, si riportano qui di seguito i menzionati articoli e il successivo articolo 98, che vanno a costituire i "Diritti relativi al ritratto", ad oggi ancora in vigore. Art. 96.: «1. II ritratto di una persona non può essere esposto, riprodotto o messo in commercio senza il consenso di questa, salve le disposizioni dell'articolo seguente. 2 . Dopo la morte della persona ritrattata si applicano le disposizioni del secondo, terzo e quarto comma dell'art. 93». Art. 97.: «1. Non occorre il consenso della persona ritrattata quando la riproduzione dell'immagine è giustificata dalla notorietà o dall'ufficio pubblico coperto, da necessità di giustizia o di polizia, da scopi scientifici, didattici o culturali, quando la riproduzione è collegata a fatti, avvenimenti, cerimonie di interesse pubblico o svoltisi in pubblico. 2. Il ritratto non può tuttavia essere esposto o messo in commercio, quando l'esposizione o messa in commercio rechi pregiudizio all'onore, alla riputazione od anche al decoro nella persona ritrattata». Art. 98.: "1. Salvo patto contrario, il ritratto fotografico eseguito su commissione può, dalla persona fotografata o dai suoi successori o aventi causa, essere pubblicato, riprodotto o fatto riprodurre senza il consenso del fotografo, salvo pagamento a favore di quest'ultimo, da parte di chi utilizza commercialmente la produzione, di un equo corrispettivo. 2. II nome del fotografo, allorché figuri sulla fotografia originaria, deve essere indicato. 3. Sono applicabili le disposizioni dell'ultimo comma dell'art. 88». 
cessitate exigente ${ }^{47}$.

Le fattispecie che, decise fra 1902 e $1907^{48}$ vedono protagoniste due giovani, Claudia Maga ${ }^{49}$ e Ida Biliotti ${ }^{50}$, e quest'ultima in particolare, accendono i riflettori sul tema del consenso, seppur differentemente declinato, e sui nessi fra lesione dell'onore e diffusione dell'immagine personale, nella prospettiva del risarcimento per lesione di diritti, la natura dei quali è di faticosa definizione. Ancor più, si nota che le argomentazioni spese dai giudici in un contesto normativo che all'epoca non consente la possibilità di riferimento ad alcuna disposizione in materia di diritti d'autore, alimentano il fitto intrecciarsi delle posizioni della dottrina intorno agli aspetti più teorici e sistematici: Eduardo Piola Caselli, Mario Ricca-Barberis $^{51}$, Valerio Campogrande ${ }^{52}$, Luigi Ferrara, Bartolomeo Dusi ${ }^{53}$ si esprimono in merito dalle pagine delle riviste periodiche e in brevi ma condensati saggi.

È subito chiaro che l'evoluzione tecnica e degli strumenti fotografici, così come

${ }^{47}$ Ricca-Barberis, 1903, p. 201 (anche per la citazione in titolo di paragrafo).

${ }^{48}$ Riporta le pietre miliari, per così dire, dell'attività dell'interprete nazionale Ferrara, 1942, pp. 83 ss., menzionando quale primo esempio di riconoscimento del diritto sull'immagine da parte della giurisprudenza italiana la decisione della Corte d'Appello di Milano del 24 aprile 1895 (in Giurisprudenza Italiana, 1895, I, 2 p. 261) per il caso di un pignoramento effettuato nei confronti di uno scultore ed eseguito sopra una statua di marmo che, ritraendo il committente, non è ritenuta nella disponibilità dello scultore (Ferrara, 1942, pp. 83-84).

${ }^{49}$ Corte App. Torino, Maga c. Cominetti, ud. 3 marzo 1903, in Foro Italiano, 1903, 28, cc. 633-645 (con nota di Piola Caselli, che di fatto è un capitolo del Trattato del diritto d'autore, in uscita per i tipi Marghieri); anche in Giurisprudenza italiana, 1903, cc. 716 ss. e riferita in Monitore dei Tribunali, 1903, pp. 99.

${ }^{50}$ La decisione Trib. Milano, 27 maggio-5 giugno 1903, Biliotti c. Varischi, Artico \&Co., è in Foro Italiano, 1903, 28, cc. 1123-1126, Giurisprudenza italiana, 1903, I ,2, cc. 713-716 e anche Rivista del diritto commerciale, 1903, II, pp. 440 ss. con attenta nota di Mario Ricca-Barberis.

51 II torinese Mario Ricca Barberis (1877-1959), laureatosi con Giampietro Chironi, studia in Germania e diviene professore ordinario di diritto civile e diritto processuale civile, esercitando la docenza nell'Università di Torino sino all'uscita di ruolo, per ben venticinque anni. Nella sua produzione scientifica, ove si distingue per essere attento commentatore della casistica giuridica, emergono proprio gli studi, numerosi e stimolanti, in tema di diritto all'immagine.

${ }^{52}$ Sull'impegno del Campogrande intorno, più in generale, al diritto sulla propria persona e alla formula del jus in se ipsum che fortuna ha in dottrina e pure in giurisprudenza, cfr. Testuzza, 2019, pp. 807 ss. (cfr. anche Caravale, 2016, p. 150) e Alpa, Resta, 2019, pp. 206 ss..

53 Il giurista veneto Bartolomeo Dusi (1866-1923), che succede a Chironi alla cattedra di diritto civile dell'ateneo torinese, dal 1918, è un civilista il cui percorso accademico piuttosto travagliato si unisce a un profilo scientifico peculiare, considerata la vocazione per gli studi romanistici e pandettistici e al contempo l'assidua collaborazione con un periodico "avanguardista" come la Rivista del diritto commerciale (cfr. Calvo, 2013). 
i nuovi media, hanno «spinto le quistioni al di fuori del campo contrattuale», giacché

[l] diffusione della stampa periodica, la tendenza, che essa rappresenta, di spandere luce di pubblicità sempre maggiore sulla vita individuale e collettiva, l'indirizzo positivo della scienza e realistico della letteratura, l'applicazione dell'arte alla multiforme réclame industriale e commerciale, rendono, d'altro canto, grave e delicato il problema sui limiti che il diritto deve imporre alla riproduzione dell'immagine affinché non siano turbati o lesi gli interessi della personalità umana ${ }^{54}$.

Se ne ha diretta percezione attraverso la casistica giudiziaria, che resta a fungere da prezioso precedente nelle argomentazioni dei giudici sino all'avvento del Codice.

La vicenda Maga, nella quale per la modella di nudo effigiata, minorenne, il padre avanza domanda di risarcimento danni da responsabilità civile, muove dalla contestazione di fattispecie penali di corruzione di minorenne e diffamazione. Appurata la professione della giovane, esercitata presso accademie e studi privati col consenso dei genitori, l'esposizione della fotografia nel contesto di una mostra d'arte non è giudicato offensivo della morale pubblica, né tantomeno di quella della minorenne, che deve già ritenersi aver perduto «quel sentimento delicato di pudicizia individuale che forma il fiore più puro e prezioso che possa adornare una donna» ${ }^{55}$. Ma scartati i connotati delittuosi dei fatti, l'attenzione è comunque focalizzata dalla Corte torinese sul danno colposo alla reputazione di giovane e familiari e, incidentalmente, anche sull'esigenza di un consenso specifico alla esposizione e diffusione dell'immagine, comunque escludendosi la proprietà delle lastre fotografiche in capo alla ritrattata.

Ancor più nitido e denso di questioni di diritto è il caso portato alla cognizione del Tribunale di Milano, nel novembre 1902, da Ida Biliotti avverso la ditta Varischi, Artico \&Co. per l'arbitraria pubblicazione e vendita sul territorio nazionale di cartoline illustrate con la sua effigie. La richiesta di risarcimento per i danni materiali e morali discende pur sempre da ragioni reputazionali: la diffusione dell'immagine mette la Bilotti «nel rango delle cosiddette bellezze professionali» e dissuade dalle intenzioni matrimoniali «il di lei fidanzato, persona ricca e dabbene».

La decisione del tribunale meneghino va tuttavia dritta alle questioni giuridiche, non indugiando sulle coloriture morali, con ciò riscuotendo l'apprezzamento della dottrina per la sua efficacia argomentativa ${ }^{56}$. II fugace richiamo al feticcio di

\footnotetext{
${ }^{54}$ Piola Caselli, 1903, p. 634.

${ }^{55}$ Corte App. Torino, Maga c. Cominetti, ud. 3 marzo 1903, in Foro Italiano, 1903, 28, c. 637.

${ }^{56}$ La nota di Mario Ricca-Barberis alla decisione del Tribunale di Milano apprezza particolarmente i riferimenti pandettistici del giudicante ed esalta il merito (non condiviso da altri autori: cfr. per esempio le caustiche osservazioni di Luigi Ferrara, sulle quali torneremo
} 
Windscheid e alla "teorica romana del precario» (qui nel senso di una forma di 'locazione' del proprio corpo), unitamente al concetto di immagine come "estrinsecazione del diritto nel proprio corpo, essendo quella riproduzione di questo» portano conseguentemente al riconoscimento del «diritto incondizionato di disposizione» della propria immagine. Tale diritto prende forma giuridica nell'obbligo del consenso espresso e revocabile alla riproduzione e diffusione, anche in caso di persona pubblica o in qualche modo notoria.

Poco tempo dopo, anche Raffaella Pecchioli ${ }^{57}$ avanza domanda per risarcimento danni nei confronti della medesima ditta, peraltro in relazione a un caso che presenta significative analogie con quello di Claudia Maga: anche la Pecchioli è minorenne (ma ultrasedicenne, dettaglio importante per la decisione di secondo grado), e si ripropone dunque il tema del valido consenso per una fattispecie di pubblicazione e diffusione, sotto forma di cartoline, dell'immagine personale. La corte milanese considera ormai ammesso dalla giurisprudenza, nazionale e ancor prima internazionale, e dalla dottrina (pur rilevando difformità di teorie), «il diritto alla propria figura», sulla scorta di uno jus in se ipsum, per il quale il diritto alla propria immagine "altro non è che una esplicazione del diritto sul proprio corpo».

Al di là del fondamento teorico, ci interessa rilevare, qui, da un profilo del processo storico di definizione della fisionomia del diritto di cui ci occupiamo, l'espresso richiamo al diritto pubblico, giustificato dalla 'non patrimonialità' del bene-immagine. Passando così dalla strada dell'onnipresente diritto alla tutela dell'onore che, come il diritto all'immagine, «rampolla dal ceppo dei diritti della personalità», è compiuta un'ardita analogia, invero un salto di specie, giacché si attinge al diritto penale, nel silenzio della legge civile. E dunque, conclude il giudice, come è possibile la querela per l'ultrasedicenne in caso di lesioni dell'onore, e come il codice penale non vieta atti di disposizione del corpo dopo i sedici anni, così il consenso di una minore ultrasedicenne ad essere fotografata (ovviamente in caso di consenso non carpito con l'inganno) deve ritenersi valido anche ai fini civili in un caso che riguardi la sua immagine.

Gli argomenti capziosi della Corte, fuorviata da appigli teorici un po' semplicistici e da una fraintesa natura esclusivamente 'morale' del diritto sull'immagine, sono immediatamente segnalati dalla dottrina che, evidenziate le forzate analogie $^{58}$, ne contesta gli esiti e ribadisce l'importanza di un consenso valido secondo

nel § 3.4.) della dottrina tedesca nella «affermazione scientifica» del diritto sull'immagine (Ricca-Barberis, 1903, p. 440), pur riconoscendo la rilevanza della giurisprudenza 'apripista' originatasi in Francia.

${ }^{57}$ Corte App. Milano, 26 luglio 1904, Pecchioli c. Varischi e Vercelli, in Foro Italiano, 1904, 30, cc. 97-101, e Rivista del diritto commerciale, 1904, II, pp. 382 ss. con nota di Ricca Barberis e, per la pronuncia della Cassazione di Torino, Foro Italiano, 1907, 32, I, cc. 10131016, anche in Rivista del diritto commerciale, 1907, pp. 431 ss. con nota di Dusi, che sostanzialmente riprende i concetti già espressi in Dusi, 1906.

58 In Ricca-Barberis, 1903 si sostiene in particolare: «altro è l'affermazione di un diritto, 
i criteri proprii del diritto civile, e specifico. Un consenso che, essendo atto di disposizione di sé stessi, è di fatto una mera tolleranza e, pertanto, è revocabile in ogni momento. I punti critici sottolineati dai commenti alla decisione di primo grado non sono né sofistici né balzani, e di fatto sono abbracciati dalla Suprema Corte che, adita dai fiduciosi legali della Pecchioli, ribalta l'esito di secondo grado, col plauso, questa volta, dei commentatori ${ }^{59}$.

Alle soglie di nuove riforme normative, un'acuta decisione del Tribunale di Roma del 1924, dunque assunta sempre in assenza di riferimenti normativi dalla disciplina del diritto d'autore, si propone di decidere su una domanda di inibitoria e risarcimento danni per la riproduzione non autorizzata di immagini personali, applicando i «principi generali di diritto», una clausola sulla quale negli anni Venti si è ridestato anche l'interesse della dottrina ${ }^{60}$. Schierandosi per il riconoscimento del diritto all'immagine quale diritto assoluto e indipendente, con un valore sia morale che patrimoniale, per conseguenza o estrinsecazione del più generale diritto sul proprio corpo, è così affermata la inderogabilità della sua tutela anche rispetto a chi, per circostanze o professione (nel caso, trattasi di un'attrice cinematografica), sia esposto alla curiosità del pubblico ${ }^{61}$. Incidentalmente, il giudice capitolino riconosce una questione per la quale non constano precedenti: oltre all'immagine si intuisce un tema d'identità personale. Pur sempre ancorata al nome e all'effige personale, l'identità evocata in sentenza non ha tanto a che fare con l'identificazione anagrafica del soggetto, quanto piuttosto con la identificazione della sua "effettiva personalità», rispetto alla quale un'errata attribuzione dei connotati può suscitare ingiuste ambiguità ${ }^{62}$.

Intanto, mentre la giurisprudenza «continua serenamente nella sua pratica ed importante elaborazione di questo diritto della personalità ${ }^{63}$ esplorandone an-

altro la tutela della sicurezza generale della consociazione» e ancora «altro è concedere il proprio corpo e posare davanti al fotografo (e fin qui corre l'analogia) altro è permettere la divulgazione del proprio ritratto al momento della sua esecuzione» (ivi, pp. 115-446). ${ }^{59}$ Dusi, 1907, p. 431.

${ }^{60}$ Sulla rinnovata e feconda attenzione per la clausola generale, restano fondamentali le pagine di Sciumè, 2002, pp. 220 ss.. Si veda anche infra, il § 3.4.2., per ulteriori spunti.

${ }^{61}$ Trib. Roma, 24 maggio 1924, Farnesi c. Pinto e società Industriale Fototecnica, in Foro Italiano, 1924, 49, I, cc. 675-679.

${ }^{62} \mathrm{Nel}$ caso di specie, all'attrice viene attribuito il nome di altra 'artista' ai fini di una réclame, cosi compiendosi «atti che possano ingenerare equivoci sulla altrui identità personale, perché [...] con l'attribuzione alla immagine del nome di altra artista si espone la persona fotografata a perdere, a favore di quella il vantaggio che dalla pubblicità legittimamente dovrebbe, se mai, a lei soltanto derivare e perché chi conosce la persona effigiata vedendone l'immagine divulgata con altro nome può ritenere essere questo un nome di battaglia assunto per ragioni non onorevoli» (ivi, c. 679).

${ }^{63}$ Ferrara, 1903, cc. 308-309. Per ulteriore casistica, anche relativa a fattispecie che riscuotono scalpore nell'opinione pubblica, in quanto coinvolgenti personalità note, ovvero che evidenziano le nuove potenzialità lesive dello sfruttamento commerciale dell'im- 
che i risvolti patrimoniali, l'attenzione dottrinale va convergendo sulla collocazione sistematica del diritto sulla propria immagine, ove ammesso ${ }^{64}$. Entrando in rapporto dialettico con un più generale 'diritto della personalità', esso, ora è riconosciuto quale mera epifania di tale diritto ${ }^{65}$, ora riesce ad emergere in quanto diritto autonomo ${ }^{66}$; le oscillazioni sono tuttavia evidenti.

II processo attraverso il quale le fucine della giurisprudenza e della dottrina sortiscono materiali d'uso interpretativo e riuso normativo prosegue per tutto il quindicennio successivo alla prima legge sul diritto d'autore ${ }^{67}$. E una diversa prospettiva va prendendo piede in particolare nella giurisprudenza: «al disopra» del diritto sull'immagine c'è una «speciale esplicazione del diritto generale della li-

magine, si veda Ferrara, 1942, pp. 92-102.

${ }^{64}$ È il caso, fra i più eclatanti, di Piola Caselli, per il quale rimando a Piola Caselli, 1903, pp. 196 ss. e Piola Caselli, 1927, pp. 306: «Il giurista che voglia lealmente costruire il sistema sulla base delle nuove regole del nostro Diritto positivo, deve, perciò, escludere che in Diritto italiano esista il cosiddetto "diritto sull'immagine propria" che l'eccessiva astrazione analitica della dottrina ha voluto immaginare». Ancora una conferma in Piola Caselli, 1943, pp. 505-507.

${ }^{65}$ Dusi, 1906, p. 216: «là [nelle leggi sul diritto d'autore] la persona viene protetta in quanto ha, qui [nel diritto sulla propria immagine] in quanto è», e dunque, il diritto alla propria immagine non ha «dignità di un diritto subiettivo per sé stante», ma è "forma di apparizione del più ampio diritto della personalità». Si vedano anche Fadda e Bensa, che nella rinomatissima traduzione delle Pandette del Windscheid, sin dalla prima versione delle Note riconoscono il diritto all'immagine e lo collocano fra i diritti della personalità (cfr. infra § 3.4.1.)

${ }^{66}$ Ne sono esempio il Ferrara, nei suoi vari interventi, e Campogrande, pur assai diversi nelle ragioni poste a fondamento della medesima conclusione.

${ }^{67}$ Le sentenze degli anni Trenta riflettono il mutare dei tempi e delle tecniche, dalle cartoline alla cinematografia, ma ancora si richiamano le decisioni dei primi del Novecento per esplicare la portata dell'articolo 11 della legge sui diritti d'autore, in tema di assenso esplicito alla pubblicazione delle immagini e specifico rispetto agli usi dell'immagine. Si veda Corte App. Milano, 21 luglio 1933, Rizzoli S.A. c. Orsini, in Rivista del diritto commerciale, 1933, II, pp. 485 ss., per un caso di impiego dell'immagine personale in una réclame piuttosto sconveniente di un prodotto industriale, oppure la vicenda decisa dal Trib. Milano, 11 luglio 1936, Lombardo c. Campari, in Monitore dei tribunali, 1937, pp. 537-538, ove si rimarca che l'assenso per l'impiego dell'immagine per uno specifico scopo non lascia supporre un'autorizzazione all'uso per altri scopi; nello stesso senso, Trib. Milano, $1^{\circ}$ dicembre 1938, Betti c. Calzificio di Vedano e Agenzia Schostal, in // diritto di autore, 1938, pp. 501 ss.. Ancora, sono portate alla cognizione del giudice vicende in cui, capovolgendo la consueta prospettiva, è l'autore a lamentare l'illecita riproduzione o cessione dell'opera a terzi da parte del ritrattato (si vedano a titolo d'esempio Trib. Milano, 17 febbraio 1936, Santacroce e Mannini c. Ditta A. e G. Garisch e C, in Monitore dei Tribunali, 1937, pp. 189190), oppure a rivendicare la separatezza del diritto dell'autore, anche morale, da quello sull'immagine e, dunque, la possibilità di agire per contraffazione o usurpazione della paternità, a prescindere dal mancato consenso dell'effigiato alla pubblicazione dell'opera (Corte Cass., 27 marzo 1929, in Giurisprudenza Italiana, 1929, II, p. 138). 
bertà che gli Inglesi designano con l'espressione di "diritto della intimità" o "right of privacy"», mentre solo nei casi più gravi "è possibile ch'esso consista nel diritto alla difesa del proprio onore personale» ${ }^{68}$.

\subsection{Fardelli d'onore}

La lunga lotta che il diritto all'immagine ha dovuto sostenere per differenziarsi dal diritto all'onore si è conclusa vittoriosamente anche nella nostra legge: il diritto all'immagine non è assorbito nel diritto all'onore ${ }^{69}$.

L'esultanza è post-codificatoria, ma i nessi col "diritto all'onore", come abbiamo visto, sono un altro laccio (o fardello) teorico, dal quale il diritto all'immagine stenta ad affrancarsi; almeno sino al progetto preliminare e ai dibattiti suscitati in commissione parlamentare, la percepita sovrapponibilità - seppur parziale - dei beni giuridici protetti persiste ${ }^{70}$ anche nella lettura dell'interprete ${ }^{71}$, soprattutto sulla scorta di certa dottrina tedesca (mi riferisco in particolare al Von Blume ${ }^{72}$ ) che ampia diffusione ottiene pure in Italia. Lo stesso Piola Caselli propende per una valorizzazione dell'esigenza di tutela dell'effigiato solo in caso di lesione dell'onore ${ }^{73}$, poiché è di norma innocua l'esposizione al pubblico della propria immagine, fatto che di per sé «non lede alcun interesse, né tocca la sensibilità di alcun tipo di persona fisiologicamente equilibrata ed educata alle esigenze della vita sociale ${ }^{74}$.

${ }^{68}$ Piola Caselli, 1927, p. 307. Si vedano anche le interessanti note di Ferrara, 1938, pp. 513 in particolare e 1942, pp. 133 e 352 ss..

${ }^{69}$ De Cupis, 1959, p. 262.

${ }^{70}$ Dico "almeno sino», in quanto giuristi del calibro di Santoro-Passarelli, Funaioli e Messina ancora negli anni Cinquanta del secolo ventesimo assorbono integralmente il diritto all'immagine nella tutela dell'onore (cfr. ivi, pp. 262-263) o, come nel caso di Pugliese, seppur da diversa prospettiva, riconducono alla lesione dell'onore la possibilità di tutelare il 'preteso' (negato dall'Autore, in quanto asseritamente fondato su assiomi moralistici o sociologici: Pugliese, 1954) diritto alla riservatezza (cfr. De Cupis, 1954, c. 90). Ma l'esigenza di "vivere in pace la propria vita» non può avere a che fare col decoro e l'onore, secondo Musatti, 1954, c. 186.

${ }^{71} \mathrm{Si}$ veda anche, più ampiamente sulle connessioni fra immagine del corpo, diritto e moralità, Fusar Poli, 2020a, pp. 261-263 e Fusar Poli, 2020b.

72 Per Wilhelm Von Blume (1867-1827), eventuali riproduzioni dell'immagine possono al più recare pregiudizio all'onore del fotografato, e dunque neppure si pone l'esigenza di ragionare di nuove disposizioni di legge o di "escogitare un nuovo diritto» (von Blume, 1903, cc. 724-725); contra Ferrara, 1903 e Campogrande, 1904, c. 920 che ammonisce: "con ciò si confondono insieme diritti di natura differente». Eppure «molti scrittori hanno negato un diritto all'immagine come un diritto a sé stante, considerandolo piuttosto come uno degli aspetti del diritto all'onore» (Degni, 1939, p. 200).

73 Piola Caselli, 1903, cc. 643-644.

${ }^{74}$ Ibidem, e ancora: «il ritratto, infatti, non è che un sostitutivo, creato dall'arte, della vi- 
È sufficiente richiamare le vicende discusse nelle aule giudiziarie in avvio del Novecento, ampiamente commentate sulle riviste specializzate, per cogliere in modo esemplare le interferenze fra i diversi piani sui quali l'immagine della persona può assumere rilevanza da un profilo giuridico, e per apprezzare, strada facendo, anche qualche commistione fra norme di diritto penale e illecito extracontrattuale.

Certo, la legge sul diritto d'autore del 1925 contribuisce a diradare la foschia che avvolge istituti e teorie, ma persiste una sorta di dicotomia, che affiora sia nella dottrina che nella giurisprudenza ${ }^{75}$, fra la soluzione che configura un diritto all'immagine dalla portata generale, con alcune eccezioni (di fatto quelle contemplate dalla legge speciale) e la sussunzione della tutela giuridica dell'immagine sotto quella del diritto all'onore della persona effigiata, con una dissoluzione del bene giuridico tutelato entro la proiezione sociale dell'immagine personale, ovvero, appunto, entro la sfera della reputazione e onorabilità.

La contrapposizione emerge dalle osservazioni sin qui svolte: una costante dialettica che va stemperandosi con il prevalere del riconoscimento di un nuovo diritto soggettivo, riflesso di una concezione che si fa strettamente personale ed intima (individualistica, per usare un attributo impiegato con accento spregiativo dalla dottrina avversa) dell'immagine, segnando piuttosto la via verso il delinearsi di un diritto alla riservatezza, sempre più omnicomprensivo e per il quale, semmai, «attraverso l'immagine è protetto anche l'onore della persona» ${ }^{76}$.

\subsection{Lunghe costruzioni}

«Ma razionalmente - si chiede Luigi Ferrara in avvio di secolo ventesimo - è fondata, per logica giuridica, la esistenza del diritto sulla propria immagine? ${ }^{77}$. Le fatiche interpretative dei giudici, i cui argomenti lasciano intuire il dibattito dottrinale in corso, giustificano l'urgenza del tema. Tanto più che il contributo della scienza giuridica è al momento della (retorica) domanda, un «mare magnum di opinioni svariatissime, non sempre giuridiche, e talvolta persino ridicole, rimescolate e agitate recentissimamente nella raffica dottrinale che intorno alla questione ha imperversato in Germania» ${ }^{78}$.

sione diretta, e la pubblicazione del ritratto non può, quindi, essere lecita quando illecita sarebbe quella visione diretta che esso sostituisce» (ivi, c. 645).

${ }^{75}$ L'art. 11 della legge sui diritti d'autore richiede conferme in tal senso: si veda la già citata decisione del Tribunale di Milano, 11 luglio 1936, Lombardo c. Campari. Ivi è perentoriamente sancita la possibilità di risarcimento in caso di pubblicazione senza consenso della persona fotografata, a prescindere da qualsivoglia lesione all'onore.

${ }^{76}$ Più in generale sulla tutela dell'onore inteso come dignità personale, ove proiettato nel diritto civile: cfr. De Cupis, 1959, p. 281 pp. 229 ss., che ricostruisce la protezione dell'onore come «limite delle limitazioni» al diritto sulla propria immagine personale.

${ }^{77}$ Ferrara, 1903, c. 302.

78 Ibidem. 
In quegli anni, Bartolomeo Dusi ${ }^{9}$ rivolge uno sguardo retrospettivo al diritto sull' immagine e vede lo "spiritualizzarsi» della sua costruzione giuridica», ovvero un processo che trae origine dalla variegata (e instabile) teorica del dominio o proprietà sul corpo e sulle sue espressioni e manifestazioni, e da lì si diparte, immergendosi nell'alveo dei diritti della personalità, categoria dalla dogmatica e dai contenuti invero ancora piuttosto incerti ${ }^{80}$.

Decenni più tardi, al tempo del Codice, potrà ormai affermarsi che

l'amplissima categoria dei diritti della persona, intanto ha potuto fare i suoi meravigliosi progressi moderni, in quanto ha costituito come un grande fondo di riserva e, si potrebbe dire quasi, di acclimatazione ed elaborazione delle specifiche nuove esplicazioni concrete della personalità umana ${ }^{81}$

e fra tali esplicazioni rientra stabilmente anche il diritto all'immagine.

Quest'ultima citazione è tratta dalla erudita voce enciclopedica in tema di diritti della persona, curata nel 1939 da Massimo Ferrara Santamaria per il Nuovo Digesto Italiano. Una voce che il giovane assistente presso la regia università di Napoli costruisce come un'appassionata perorazione della categoria dei 'diritti della persona'. Nello sforzo sistematico profuso sulle colonne della prestigiosa enciclopedia sono riallacciati i nodi con la dottrina tedesca di fine Ottocento e primi Novecento (maneggiata con disinvoltura tale, da non lesinare critiche ai suoi più eminenti rappresentanti) e con la riflessione antiformalista europea, e sono rimeditate le più recenti riflessioni della scienza giuridica nazionale, che ai medesimi riferimenti esteri attinge, pur imprimendovi una propria distinguibile impronta.

Ora, non può certamente essere questa la sede per una digressione che segua il lungo e tortuoso percorso dei diritti della personalità, al quale molteplici affluenze teorico-dogmatiche hanno portato alimento in prospettiva storico-giuridica ${ }^{82}$. L'impresa può tuttavia opportunamente ridimensionarsi, entro la prospettiva d'analisi di questo studio, muovendo dall'angolo visuale del diritto all'immagine, attraverso le fonti che abbiamo sin qui assunto quali strumento e oggetto d'indagine, e osservando lo sguardo - mi si passi il jeu de mots - di chi tale percorso ha vissuto, partecipandovi in prima persona.

\footnotetext{
${ }^{79}$ Dusi, 1906, p. 211 e 1907, p. 437.

${ }^{80}$ Incerta sin dalla formula lessicale "diritti della personalità": Ferrara Santamaria, 1939, p. 914 insiste, al di là delle denominazioni prescelte in dottrina, sulla sostanza della categoria, che include "diritti essenziali e indissociabili dal loro genuino originario soggetto», per un «evidente e naturale vincolo o rapporto d'inerenza tra la persona e certe sue personalissime esplicazioni di attività e libertà». La teoria del "rapporto d'inerenza" è sviluppata più ampiamente in Ferrara Santamaria, 1937b.

${ }^{81}$ Ferrara Santamaria, 1939, p. 925.

82 Per riferimenti bibliografici, principalmente offerti dalla letteratura giuscivilistica, cfr. supra, nota n. 3.
} 


\subsubsection{Giusnaturalismi e "tedescherie"}

I diritti della personalità, a cui la dottrina decisamente prevalente intorno alla metà del Novecento ascrive il diritto sull'immagine, sono pervenuti al pensiero giuridico moderno

attraverso l'antica concezione giusnaturalistica dei diritti innati ed il successivo sforzo della teoria, inteso a distaccare dalla personalità alcuni diritti costituenti la sua prima ed essenziale manifestazione ${ }^{83}$.

Lo rileva anche Maroi, nel Commentario al Codice diretto da Mariano D'Amelio, evocando ( $m a$ il richiamo è già presente nei lavori di codificazione), quale prima mitologica estrinsecazione normativa di queste origini, il $\S 16$ dell'ABGB ${ }^{84} \mathrm{e}$ il concetto dei diritti innati ivi adombrati quale nucleo essenziale della persona. Alla scuola tedesca e a quella italiana è poi toccato rispettivamente il compito di «isolare la personalità dall'uomo» e precisare il fondamento "della personalità come qualifica giuridica» e, di qui, i diritti della personalità ${ }^{85}$. Una ricostruzione semplificata e accelerata, che sfocia conclusivamente nelle disposizioni del nuovo Codice civile, ove ogni individuo, «in quanto membro della comunità giuridica» (e fatta salva la temporanea condizione dell'appartenenza alla razza ariana)

ha diritto verso lo Stato ad una particolare protezione adversus omnes della sua persona fisica (vita, integrità del corpo), dei suoi attributi (nome, pseudonimo, immagine) e della esplicazione delle sue energie fisiche e spirituali.

Dalle prime identificazioni dei 'diritti innati', alla reazione fascista avverso la «aberrante» teoria del diritto sul proprio corpo, quintessenza di un rinnegato individualismo giuridico dal quale si temono derive disgregatrici ${ }^{86}$, il percorso è complesso, fitto di ramificazioni e innesti.

Anzitutto, considerando i giuristi del primo Novecento, notiamo che tentano di porre un poco di ordine, di dominare in qualche modo le direzioni di sviluppo di tale percorso, uscendone disorientati. Si trovano anche nelle decisioni delle corti nazionali tentativi di richiamare il frammentato quadro teorico di riferimento, con tanto di scelte di campo fra adombrate opposte concezioni, ma se ne coglie

\footnotetext{
${ }^{83}$ De Cupis, 1959, p. 3. Sui diritti innati attraverso il prisma della proprietà intellettuale, cfr. Moscati, 2020, pp. 194 ss. e passim, nonché Bertani, 2011, pp. 235-238.

${ }^{84}$ In merito al connotato giusnaturalistico che innerva la codificazione austriaca, e al suo portato successivo, rimando alle osservazioni di Ferrante, 2006 (per una diversa lettura, cfr. Welser, 2015, pp. 18-21) e Caroni, 1996, pp. 63-64; in generale sulla codificazione austriaca e l'apporto di Karl Anton von Martini, menzionato espressamente anche da legislatore novecentesco e dottrina, si vedano Cavanna, 2005, 272 ss. e Cassi, 1999, passim. ${ }^{85}$ Maroi, 1940, p. 84 (con chiari riferimenti a Dusi, 1906, per il quale, della personalità l'immagine è «misteriosa e quasi divina impronta»).

${ }^{86}$ Il lessico è quello di Maroi, 1940, p. 97, in tema di «Atti di disposizione del proprio corpo".
} 
più che altro un uso superficiale e retorico, incalzato dalla lacuna legis.

Certo, nei primi due decenni del secolo ventesimo la dottrina tedesca, filtrata dalla scienza giuridica italiana, è riconosciuta protagonista della scena: le note e commenti a sentenza la chiamano a sostegno e talora la sviscerano, offrendo un interessante spaccato dei riferimenti dominanti. La primazia della civilistica tedesca è ascritta anzitutto a Hugo Keyssner e al suo Das Recht am eigenen Bilde del $1896^{87}$, sebbene più incisivi appaiano i successivi apporti di Karl von Gareis, Josef Kohler ${ }^{88}$ e Otto von Gierke. Pur nelle diverse declinazioni dei loro contributi, vi si riconosce un primo effettivo sforzo di elaborazione di un fondamento dei diritti della personalità, seppur con esiti contraddittori.

A fronte di qualche convinta attribuzione di merito, la presunta ascendenza tedesca suscita anche qualche fastidio: simili «tedescherie» ${ }^{89}$ concettose e di stampo rigidamente romanistico non possono elidere il determinante e pragmatico apporto francese, più risalente, e l'autonomo e costruttivo impulso teorico italiano, a partire dalle note di Carlo Fadda e Paolo Emilio Bensa al libro secondo delle Pandette del Windscheid ${ }^{90}$. La originaria riflessione del pandettista tedesco sui diritti soggettivi e sui beni incorporali, che peraltro non contempla un diritto all'immagine, è effettivamente aperta e sviluppata dai due traduttori italiani $i^{91}$, sino a configurare un primo ed emblematico approccio sistematico alla comples-

${ }^{87}$ Per un'accuratissima ricostruzione della dottrina tedesca in tema, cfr. Resta, 2007, pp. 1045 ss., ma si veda anche Ferrara, 1942, pp. 134-154, con specifico riferimento alla riflessione che concerne il diritto all'immagine.

88 Josef Kohler (1849-1919), tradotto in Italia da Raffaele Majetti (Grossi, 2000, p. 104105) e Ferruccio Foà, è affrontato in relazione alla industrialistica italiana, in Fusar Poli, 2012, pp. 88-89 e Mazzarella, 2007, passim; entro la letteratura tedesca, sugli apporti di Kohler in tema di geistiges Eigentum, segnalo altresì Adrian J., Nordemann W., Wandtke A.A., 1996.

${ }^{89}$ Ferrara, 1903, cc. 308 ss..

${ }^{90}$ Ivi, c. 305 (e Ferrara, 1942, pp. 123-124): la loro è la «vera delineazione giuridica, precisa e incisiva, del diritto sulla propria immagine», da ricondursi dunque, cronologicamente agli anni Novanta dell'Ottocento, ovvero alle prime uscite delle Note (cfr. nota seguente). ${ }^{91}$ Sulle connessioni fra il contenuto romanistico delle res incorporales e quello mediato dalla pandettistica di cui Fadda e Bensa ci forniscono ampie prove proprio affrontando i nostri temi, cfr. Turelli, 2013. Sappiamo che le dispense con la traduzione dell'opera, che nel frattempo Windscheid va aggiornando per la settima volta, e con le preziose annotazioni di Fadda e Bensa iniziano a circolare dal 1886 e arrivano all'edizione complessiva fra il 1902 e il 1904. Le Note, originariamente parte integrante del testo tradotto, poi divengono volume a parte (edite separatamente e proseguite da Pietro Bonfante: Fadda, Bensa, 1926, pp. 122-152 in tema di diritti della personalità), come sorta di corpo organico e in aggiornamento. Si veda in particolare la Nota e) sul $\S 40$ del Secondo libro delle Pandette. Per utili riferimenti biografici in merito a Carlo Fadda (1853-1931) e Paolo Emilio Bensa (1858-28) e ai rispettivi percorsi scientifici strettamente intrecciati fra loro, rinvio alle voci Solimano, 2013 e Notari, 2013, nonché complessivamente a Furfaro, 2016, passim. 
sa categoria dei diritti della personalità, che include, quale «esplicazione del diritto sul proprio corpo» anche il diritto sull'immagine personale ${ }^{92}$.

Proprio con riferimento al fondamento originario, che è, per così dire, 'corporale', dei 'diritti della persona' o 'della personalità', si ricercano radici ancor più remote, a muovere dalla riflessione cinque- e secentesca intorno a potestas e ius in se ipsum di Baltasar Gómez de Amescua e Samuel Stryck ${ }^{93}$, riconosciute compattamente quale battuta d'avvio, momento generativo di quel graduale concentrarsi in una figura giuridica unitaria delle varie manifestazioni del potere autonomo e incomprimibile dell'individuo sopra se stesso. Il ricorso all'attributo 'propria', unito a 'persona' (e 'immagine') in dottrina non è che un chiaro rimando lessicale a questa costruzione teorica dei diritti della personalità, imperniata su forme di titolarità - dalla proprietà al dominio, a una più generica appartenenza - della persona sul proprio corpo, le sue parti, le sue manifestazioni esteriori.

Ed è un rimando che persuade in particolare la giurisprudenza. In effetti, l'influenza sull'interprete di tali teorie (invero quali schemi argomentativi pragmaticamente semplificati) che attingono alla Scolastica secentesca così come a una Pandettistica passpartout, è apparentemente più duratura che in dottrina, dove peraltro non mancano voci assai autorevoli fortemente critiche, come quella di Adolfo Ravà ${ }^{4}$, sin da inizio Novecento. Non pare infatti fecondo il richiamo allo jus

${ }^{92}$ Si veda la nota $f$ ) sulla nota 2 al $\S 41$ (Fadda, Bensa, 1926, 152-183) e, per approfondimenti, Furfaro, 2016, pp. 317 ss. e 351-352.

${ }^{93}$ Ferrara Santamaria, 1939, p. 915 e 1937, pp. 175-176; Ravà, 1901 (che menziona anche Wolff e de Wattel: pp. 7-24); Ricca-Barberis, 1903, pp. 193-196. II riferimento è, in particolare, al Tractatus de potestate in se ipsum del giurista iberico e alla quindicesima Dissertatio del volume secondo delle Dissertationum juridicarum francofurtensium, intitolata De jure hominis in se ipsum, del giurista tedesco. Sulla riemersione e 'riattivazione' degli scritti di de Amescua al finire dell'Ottocento, cfr. Testuzza, 2016, anche con riguardo alla risignificazione della formula entro la rinnovata attenzione per il dato corporeo nella riflessione della scienza giuridica contemporanea, a partire da Enrico Ferri, Carlo Lessona, Valerio Campogrande, Adolfo Ravà (ivi, pp. 4-24, 337-354).

94 Trattasi di «concetti antichi, che i tempi nostri hanno interamente superato»: Ravà, 1901, pp. 92-93 (cfr. anche Dusi, 1907, pp. 437-438). Ravà manifesta chiaramente la sua posizione contraria alla identificazione di una categoria di 'diritti della personalità' nell'opera che, edita nel 1901, è la rielaborazione della sua tesi di laurea, su un tema sollecitato dal professor Icilio Vanni. Attingendo a strategie argomentative a contrariis, anche a Darwin, alla teosofia e alla psicologia dell'autocoscienza, e concentrandosi sulla libertà come "elemento primo» nonché sull'esigenza di separare la sfera del diritto da quella della morale, egli evidenzia la «quantità di sottigliezze e di artifici» e la confusione fra persona e personalità (termine astratto che rappresenta un «malvezzo spagnoleggiante») che connotano la prima riflessione sui diritti della personalità, e afferma l'unità della persona come subiectum juris (pp. 175 ss.). Contestati i tentativi teorici che appaiono al Ravà frutto di una confusione fra soggetto e oggetto di diritto, ovvero del ricorso inopportuno a costruzioni dogmatiche proprietarie, egli conclude che la categoria dei diritti della personalità, particolarmente in quanto fondata sul jus in se ipsum è solo «l'espres- 
in se ipsum, alla luce dei paventati sviluppi destabilizzanti di simili teoriche, che danno luogo a "concezioni diversissime e quasi opposte, comprendendo molti istituti di varia indole, e che non hanno tra loro niente a che fare ${ }^{95}$, ivi incluso il diritto all'immagine (in fondo forse fra i meno problematici ${ }^{96}$ ).

All'opposto, tuttavia, non mancano neppure voci che, ancora alle soglie del Libro primo del Codice civile, riconoscono nel processo di definizione teorica dei diritti della personalità un mero «rammodernamento secondo gli attuali criteri della sistematica moderna ${ }^{97}$, proprio della più risalente teoria dei diritti sulla 'persona propria' e, lungo questo solco, pervengono a un conclusivo e omnicomprensivo "diritto alla illesa intimità privata " ${ }^{98}$ desunto dalle argomentazioni dei giudici della più rilevante giurisprudenza nazionale, quella stessa che s'è poco fa presa in esame.

Le viscosità in dottrina sono evidenti, così come qualche aprioristica opposizione di principio.

Nell'emancipazione da ricostruzioni dogmatiche imperniate su forme di dominio, se non anche di proprietà sul proprio corpo e sulle sue parti o estrinsecazio$\mathrm{ni}$, è molto efficace il contributo della riflessioni intorno alle opere dell'ingegno, che attraverso la porta degli Immagterialgüterrechte e degli Individualrechte (con contrapposizioni interne alla stessa dottrina tedesca che li sviluppa ${ }^{99}$ ) contribuiscono ad alimentare anche in Italia «una storia di interferenze e contatti recipro-

sione giuridica di una tendenza ad un individualismo così spinto, che ha dell'anarchico. Quel credere che si possa stabilire in massima un diritto dell'uomo su se stesso, significa disconoscere che la vita sociale investe talmente l'individuo che egli le è legato in modo intimo e indissolubile» (ivi, p. 210). Afferma invece con forza la proficuità teorica del jus in se ipsum Ricca-Barberis, 1905, p. 448 ss., che si rifà ampiamente a Fadda e Bensa e alla dottrina tedesca.

${ }^{95}$ Ravà, 1901, p. 214 (piuttosto caustico sulla «bagatella di roba» rappresentata dalle elucubrazione teoriche tedesche, ivi, p. 89); parla poi di «"malattia cronica" dei giuristi tedeschi» con riferimento alle commistioni fra diritti della personalità e diritto d'autore Ferrara, 1942, pp. 203 ss..

${ }^{96}$ Ravà, 1901, p. 90.

${ }^{97}$ Ferrara Santamaria, 1937a, pp. 190-191: ivi, peraltro, I'Autore richiama proprio il pensiero di Adolfo Ravà, "non fautore dei diritti sulla persona propria», in tanto in quanto protagonista di una "consacrazione del diritto alla "riservatezza" in un'opera di carattere istituzionale», riferendosi alle sue Istituzioni di diritto privato, edite a Padova sempre nel 1937.

${ }^{98}$ Ivi, p. 183.

${ }^{99}$ Sulla teoria degli Individualrechte, distinti dagli Immaterialgüterrechte, avanzata da Josef Kohler e avversata da Otto von Gierke, da cui sarebbe derivato uno "stranissimo intruglio" con commistione, nel novero dei diritti della persona, delle tipologie di diritti più eterogenee, dal diritto alle libertà civili, al diritto sul segreto delle missive, dal diritto all'onore, ai diritti d'autore, cfr. Ferrara Santamaria, 1937, pp. 917-919 e 922; con specifico riferimento alle teorie di Kohler intorno al marchio come Individualrecht, si veda Mazzarella, 2019, pp. 872 ss. e più dettagliatamente Mazzarella, 2007, passim. 
ci $»^{100}$ fra diritti d'autore e diritto all'immagine, destinata a perdurare proficuamente, sino all'oggi ${ }^{101}$.

Il percorso d'affinamento teorico, in atto da decenni ed evidentemente connesso ad insopprimibili esigenze della realtà e a una sensibilità anche giuridica che trascende le contingenze storiche, non cede alla postura antipersonalistica del regime e trova spazio nel Codice ${ }^{102}$, da dove i diritti della personalità «sobriamente regolati» e posti in dialogo con la normativa speciale, troveranno successivi validi canali d'espansione grazie all'attività dell'interprete ${ }^{103}$.

\subsubsection{Suggestioni novecentesche fra ragione e principi}

Il ricorrere dei richiami ai diritti 'innati', cioè spettanti per natura alla persona, nonché l'espresso riferimento, diretto o mediato, al giusnaturalismo nelle costruzioni e ricostruzioni teoriche relative ai diritti della personalità e al diritto sull'immagine, ci ricordano quel fenomeno di reviviscenza del diritto naturale che riguarda l'Otto-Novecento e che si riattiva soprattutto in corrispondenza dei periodi post-bellici, quale reazione al giuspositivismo (preannunciata dall'antiformalismo), e poi anche quale risposta ai totalitarismi di metà secolo ${ }^{104}$.

\footnotetext{
${ }^{100}$ Resta, 2007, p. 1047.

101 Più in dettaglio, sul pensiero tedesco intorno ai Persönlichkeitsrechte cfr. Alpa, Resta, 2019, soprattutto alle pp. 180-193, alla cui accuratezza senz'altro rimando, sottolineando altresì la ricchezza delle fonti, dottrinali e normative, menzionate; vedansi altresì le pp. 209-211 e 219-221 per il contributo teorico offerto in tema dalla scienza giuridica nazionale in avvio di Novecento, anche attraverso il processo di definizione di un diritto morale d'autore.

${ }^{102}$ Si parla forse con troppa enfasi di chiaro «valore politico di questa soluzione legislativa» prendendo peraltro a riferimento le parole spese da Maroi sul tema nel Commentario D’Amelio (De Cupis, 1959, p. 16): la stessa fonte normativa ci svela, infatti, una ben maggiore intrusione dell'ideologia fascista nella discussione e redazione dell'art. 5 in tema di "Atti di disposizione del proprio corpo".

${ }^{103}$ La folta casistica in materia di responsabilità civile fornisce esiti interpretativi gravidi di spunti anche per la ulteriore definizione del perimetro dei beni della personalità, per il quale si vedano De Cupis, 1959, pp. 4-5; Ferrara Santamaria, 1937a, pp. 187-189: «tutto, in fondo, qui si riduce a una questione di danni e ad un'azione di accertamento per far dichiarare la violazione di questo diritto della personalità» (il riferimento è al «diritto alla illesa intimità privata» che, nella ricostruzione dell'autore, assorbe quella dei diritti della personalità: si veda in merito anche Ferrara Santamaria, 1939, pp. 923-925). Insiste sulla rilevanza dell'iniuria nel senso di «intrusione dei terzi in un campo in cui il nostro arbitrio dev'essere assolutamente ed esclusivamente sovrano» Dusi, 1907, p. 435.

${ }^{104}$ Sempre fondamentale sulla 'coppia dialettica' giusnaturalismo/giuspositivismo in prospettiva filosofica è Bobbio, 2011 (cfr. soprattutto pp. 139-170 per una riflessione critica sul diritto naturale); per esempi di riemersione di temi e letture giusnaturalistiche nel corso del Novecento, si vedano poi, Barberis, 2000, pp. 34-39; Fassò, 2006, pp. 225 ss. e 328 ss.; Viola, 2016. È un'idea di diritto naturale qui considerata è «più prossima a quella,
} 
Non mi addentro in questioni inaffrontabili qui per ampiezza e portata, quali sono quelle suscitate dal tema del diritto naturale e dalle declinazioni che esso storicamente assume, particolarmente in età contemporanea. Intendo piuttosto riservare lo spazio circoscritto di una breve digressione conclusiva a qualche spunto che mi pare possa alimentare, per via di suggestioni, una riflessione intorno alle intersezioni fra diritto naturale, principii del diritto ed elaborazione del diritto all'immagine.

Inizio idealmente a rintracciare queste connessioni richiamando il significativo peso specifico di certa dottrina tedesca non riconducibile alla pandettistica né tacciabile di formalismo giuslegalistico: penso a Josef Kohler, tradotto e apprezzato pure in Italia, anche (o forse soprattutto) proprio per la teoria dei diritti sui beni immateriali, assai rilevante da un profilo concettuale per la teorizzazione dei distinti diritti della personalità. L'acuto sguardo comparatistico, l'interesse antropologico e sociologico, la curiosità poliedrica per gli spazi giuridici più inesplorati, l'idea che il diritto si costruisca attraverso l'interazione fra norma, scienza giuridica e giurisprudenza ${ }^{105}$ sono aspetti che traspaiono dai numerosi scritti così come dai corsi universitari del giurista tedesco.

E fra le altre lezioni berlinesi, anche quelle di Kohler sono seguite da un giovane Giorgio Del Vecchio ${ }^{106}$, nel periodo di costruzione delle basi del suo pensiero anche attraverso il dialogo con le diverse scuole filosofiche tedesche a lui contemporanee. Al di là dell'effettivo incontro scientifico, certo assai formativo per il giurista bolognese, è immediata l'associazione fra questa esperienza giovanile e il suo profilo scientifico, così improntato al dialogo internazionale e alla comparazione, nonché orientato al superamento del positivismo giuridico nella sua accezione anti-filosofica e formalistica.

Oltre il fenomeno normativo, per del Vecchio esiste una «ragione giuridica naturale» che deve orientare anche l'attività dell'interprete: sono principi che nutrono l'ordinamento giuridico e che si impongono all'attenzione del giudice ove non sovviene la norma. Del Vecchio ne discute nella sua arcinota prolusione Sui principi del diritto, letta per il corso di Filosofia del diritto nella regia Università di Roma il 13 dicembre $1920^{107}$, parlando anche di corpo, personalità umana,

di origine sociologica, del diritto libero, o a quella, kantiana, della giustizia come principio formale, che non a quella sostenuta per secoli dal giusnaturalismo che abbiamo chiamato tradizionale, richiamantesi a un codice eterno e definito di norme perfette» (Fassò, 2006, pp. 335-336).

${ }^{105}$ Meccarelli, 2011, p. 728.

${ }^{106}$ Per note biografiche e approfondimenti sul pensiero filosofico di Giorgio Del Vecchio (1879-1870), si vedano Fassò, 2006, pp. 231 ss. e Montanari, 2013; interessanti elementi utili alla ricostruzione del percorso umano e scientifico di Del Vecchio anche i numerosi contributi raccolti in Bartoli, 2017.

${ }^{107}$ Enorme è la fortuna della prolusione, come noto in storiografia: ne sono curate numerose riedizioni per il mercato nazionale e internazionale (soprattutto in America Latina). Per il testo dell'intervento, si veda Del Vecchio, 1921, e anche la sua riedizione del 2017, 
libertà e volontà, e così imbattendosi nell'immagine. Mentre affronta il tema del «rispetto dovuto alla libertà, come espressione del valore assoluto della personalità umana», vero "principio giusnaturalistico» di portata generale per quanto non positivizzato, esemplifica casi di "coordinazione intersubiettiva», in cui "è perfettamente ammissibile che si determinino anche vere e proprie limitazioni dell'arbitrio individuale, non espresse nella legge, e tuttavia giuridicamente valide, perché appunto fondate nei principî generali cui la legge si riferisce». ${ }^{108}$.

Fra gli esempi di possibile ricorso ai principi generali del diritto per colmare lacune normative e bilanciare i conflitti intersoggettivi fra le rispettive libertà, Del Vecchio menziona proprio, in via paradigmatica, il diritto all'immagine:

Nessuna disposizione di legge nel diritto nostro vieta, per esempio, a un pittore o a un fotografo di ritrarre l'immagine di chicchessia, ed anche di divulgarla; né un tale divieto potrebbe ricavarsi da altre disposizioni per mezzo di analogia. [...] Ma il più generale e vero principio, che abbraccia, non solo negativamente, l'essenza della personalità umana, quel principio che è il grande sottinteso del nostro sistema giuridico, obbliga ad un esame più profondo e, vorremmo dire, più sostanziale.

Trovandosi a porre in rapporto la «volontà dell'artista di ritrarre e di divulgare l'immagine altrui» con la "volontà di colui, cui può non piacere la divulgazione delle proprie sembianze», il giurista non deve «appagarsi di riconoscere la mancanza di una legge in tale materia; dal che consegue soltanto l'inammissibilità di una restrizione d'ordine pubblico, per es. di natura penale». Egli allora può ricercare una "soluzione che segni il limite nel contrasto degli opposti voleri»,

risalendo al principio generale del rispetto della personalità umana, e ponendo in relazione con esso quella particolare estrinsecazione della personalità che consiste nella fisionomia propria di ciascuno. Sorge di qui la figura giuridica del «diritto sulla propria immagine» che si viene elaborando dai nostri giuristi, non senza

pp. 15-63 qui utilizzata, preceduta da interessanti notazioni di Bruno Romano (pp. 3-14). Sugli effetti perturbanti della prolusione sulla dottrina italiana del tempo, si veda anche Sciumè, 2002, pp. 220 ss. e, con riguardo anche alla provocazione del diritto naturale nel contesto dei dibattiti sul tema dei 'principii generali', occasionati dal ben noto convegno pisano del 1940 (se ne veda la prima stesura nel prezioso Convegno nazionale Universitario su i principi generali dell'ordinamento giuridico fascista tenuto in Pisa nei giorni 18 e 19 maggio 1940-XVIII, Pisa, 1940), cfr. ivi, pp. 212 ss..

108 "Del tutto oscura è, per esempio, nella nostra legislazione la nozione del diritto sul proprio corpo, il quale diritto è riconosciuto e protetto solo indirettamente, per mezzo delle guarentigie generali d'ordine pubblico, delle leggi penali che concernono $\mathrm{i}$ «delitti contro la persona», e in fine della legge civile che impone il risarcimento del danno in conseguenza di ogni fatto colposo. L'insufficienza di un tale riconoscimento generico e indiretto, sì per i fini scientifici come per la risoluzione dei casi pratici, è resa manifesta da ciò, che ancora da molti si nega l'esistenza di un diritto sul proprio corpo, mentre fra coloro medesimi che l'ammettono sono vivi i dissensi sulla natura e sulle conseguenze di esso» (Del Vecchio, 1921 e Fusar Poli, 2020a, pp. 253 ss.). 
pratiche applicazioni, e che suppone un fondamento di ragione nel diritto nostro, oltre gli espressi termini della legge.

Sono parole evocative di un importante momento di passaggio, che è della scienza giuridica come della giurisprudenza, entrambe impegnate nel processo di superamento delle impostazioni tradizionali, che nei primi anni Venti ancora si svolge al di fuori di una cornice normativa ad hoc. I 'principi generali del diritto' appaiono già come un prezioso diaframma (che presto si aprirà verso l'ordinamento tutto) attraverso il quale ri-definire fisionomie giuridiche e delinearne di nuove ${ }^{109}$. Dal «fondamento di ragione» che evoca la matrice giusnaturalistica e un'ascendenza neo-kantiana, al valore costituzionalmente garantito della persona umana, il diritto all'immagine richiama, e seguiterà a richiamare, la necessità «politico-sociale, oltre che etica, di adattare il diritto alla sempre più rapida trasformazione della società ${ }^{110}$.

\section{Direzioni}

Se questa fosse l'occasione per approfondire anche il secondo Novecento, potrei ora proseguire occupandomi del processo mediante il quale giurisprudenza e scienza giuridica fertilizzano il terreno normativo codicistico, dando vita a inattesi sviluppi e trasformazioni del peculiare diritto sul quale ci siamo soffermati in queste pagine.

La "funzione identificatrice» svolta dall'immagine anche oltre la sua accezione anagrafica, tradizionalmente accolta ${ }^{111}$ è uno di questi, già intuito negli anni ' 40 :

${ }^{109}$ In generale sull'evoluzione storica della formula dei principii generali del diritto, rimando a Sciumè, 2002.

${ }^{110}$ Fassò, 2006, p. 336.

111 «Il soggetto, come unità della vita sociale e giuridica, ha bisogno di affermare la propria individualità distinguendosi dagli altri soggetti e risultando per chi è realmente. II bene che soddisfa tale bisogno, è il bene dell'identità, il quale consiste precisamente nel distinguersi nei rapporti sociali dalle altre persone, risultando per chi si è realmente. Difficile è determinare se tale bene proceda, ovvero segua, nella gerarchia dei modi di essere morali della persona, i beni dell'onore e della riservatezza; ad ogni modo, che abbia grande importanza non c'è dubbio, perché l'uomo annette grande valore all'affermarsi non soltanto come persona, ma come una certa persona, evitando la confusione con le altre» (De Cupis, 1949, p. 13). Un'accezione innovativa, questa, e «di transizione», fra la lettura restrittiva tradizionale, per la quale l'interesse all'identità personale si incarna «nel diritto all'uso esclusivo dei segni della persona», e la lettura attuale, affermatasi intorno agli anni Settanta del Ventesimo secolo, che sostanzia la nozione di identità personale nella «immagine sociale che l'individuo proietta di sé in relazione al complesso delle idee, delle convinzioni, delle posizioni politiche, degli atteggiamenti culturali e di quant'altro costituisca espressione esterna del patrimonio morale di quell'individuo" (Pino, 2003, pp. 42-43 e, per una progressiva valorizzazione dell'interesse - non ancora del diritto soggettivo autonomo - all'identità come "verità personale», per arrivare a un'idea d'identità disan- 
ne Il diritto all'identità personale, progettato quale audace trattato organico in due parti da Adriano De Cupis, all'epoca professore di diritto privato all'università di Perugia, sin dal primo (che resterà unico) volume monografico su /l nome civile, l'immagine è presa in considerazione in relazione allo spettro semantico più ampio dell'identità, dunque oltre "l'angolo visuale della riservatezza personale ${ }^{112}$, entro il quale sin dagli anni Trenta, come abbiamo visto, e sempre più stabilmente è collocata la prospettiva del diritto all'immagine.

Ma negli anni Cinquanta, e ancor più dalla fine degli anni Sessanta, sulla scorta della nuova grammatica costituzionale ${ }^{113}$ cambieranno gradualmente e significativamente lessico e sintassi dei diritti della personalità, e proprio gli articoli dal 5 al 10 del Codice civile, dal quale ultimo siamo partiti per questo esperimento di scomposizione storico-giuridica, manifesteranno una grande vitalità ${ }^{114}$. L'intero novero dei diritti della personalità, da insieme eterogeneo originatosi per «accostamento di norme dalla diversa storia» potrà così progressivamente compattarsi entro una comune dimensione di senso, nel definire la quale l'apporto giurisprudenziale sarà ancora fondamentale ${ }^{115}$.

corata dal diritto al nome e all'immagine, Pino, 2003b, con ampi riferimenti alle fonti). 112 Noto come già per De Cupis, 1949 «il c.d. diritto all'immagine [...] è il diritto al riserbo nei riguardi della propria immagine, del proprio aspetto fisico così com'è percepibile visivamente» (p. 258). II diritto alla riservatezza intraprende il suo cammino sempre percorrendo i canali giurisprudenziali e assorbendo gli stimoli dalla letteratura giuridica straniera, in questo caso particolarmente anglosassone, sebbene il right of privacy appaia piuttosto estremo nella sua portata ai giuristi nazionali, più cauti nel valutare i contorni di tale nuova posizione giuridica soggettiva e inclini alle diciture nostrane, fra le quali anche «diritto di essere lasciato solo», o «diritto alle vicende» (Ligi, 1955). Invero i cenni al right of privacy appaiono sin dall'avvio del Novecento, dimostrando cosi lo sguardo attento della scienza giuridica italiana sulle riviste e sulla letteratura giuridica d'Oltreoceano, ma anche in alcune intuizioni di Kohler in tema di «diritto alla solitudine» (Ferrara, 1903, c. 203, ribadito in Ferrara, 1937b, pp. 52 e 509-514; Ricca-Barberis, 1903, p. 196; contra Campogrande, 1904, c. 922). Vale la pena notare qui, incidentalmente, il legame, denso di ispirazioni reciproche, del Kohler con la dottrina americana, particolarmente con Roscoe Pound e la Sociological Jurisprudence (si vedano anche le incursioni nelle fonti americane riportate in Kohler, 1903, pp. 43 ss.).

${ }^{113}$ Sul dialogo fra Costituzione e Codice civile, e sul fondamentale ruolo della Corte Costituzionale quale mediatrice entro tale dialogo, si veda Alpa, 2018, pp. 508 ss..

${ }^{114} \mathrm{E}$ in particolare il diritto sulla propria immagine: oltre a De Cupis, 1959, menziono, fra i capisaldi della trattatistica in materia, esemplare della non univocità dottrinale rispetto alla natura del diritto sull'immagine, Vercellone, 1959, ove piuttosto ci si riferisce a un "diritto al ritratto", o meglio, a un diritto avente per oggetto le singole rappresentazioni delle sembianze del soggetto.

${ }^{115}$ La pronuncia della Corte Costituzionale, 12 aprile 1973, n. 38 ci mostra la prima decisione della Consulta in tema di diritto all'immagine: per tale pronuncia esso appartiene ai "diritti della personalità rientranti in quelli inviolabili che la Costituzione salvaguarda, tenuto anche conto della estrema importanza di tali diritti, della gravità e dell'irreversibilità 
Lo vediamo sempre in De Cupis quando, dieci anni dopo il suo trattato incompiuto, nel contribuire al prestigioso Trattato di diritto civile e commerciale diretto da Cicu e Messineo, cristallizza lo stato dell'arte, impiegando strumenti ermeneutici e dogmatici ormai affinati, per affrontare più ampiamente la materia de $I$ diritti della personalità ${ }^{116}$ nella loro proteiforme complessità. L'ardua sistematizzazione sortisce pagine che fermano come in un'istantanea un soggetto cangiante e in rapido movimento, esposto al rapido mutare della sensibilità giuridica e sociale: sono dunque inevitabili alcune sfocature. Tuttavia, sono pagine preziose, dalle quali emerge anche un "eterno ritorno del diritto naturale» (il richiamo è anche a Del Vecchio) e la sua rilevanza teorica nella configurazione dei diritti della personalità quali diritti essenziali ${ }^{117}$.

Nel suo edificio sistematico, De Cupis colloca con sicurezza il diritto all'immagine entro la sezione dedicata a /l diritto alla riservatezza, ove accoglie il «diritto alle vicende» personali e al riserbo ${ }^{118}$, così come il "diritto al 'segreto'» nelle sue molteplici estrinsecazioni normative, e ove il diritto all'immagine diviene «diritto

del danno che la violazione di essi arreca agli interessati e che può incidere irrimediabilmente sulla loro posizione sociale e su quella dei loro congiunti, dell'impossibilità di ripararlo adeguatamente, dell'esigenza di un pronto intervento per impedire che il pregiudizio si verifichi». Inoltre, "risulta evidente che la tutela dei diritti sulla propria immagine, su quelle dei genitori, dei coniugi e dei figli [...] che venga esercitata anche rispetto alle attività strumentali e alle cose le quali siano dirette e servano all'organizzazione, predisposizione, preparazione e formazione del materiale che si presume destinato ad essere pubblicato, non trova ostacolo nel precetto costituzionale di cui all'art. 21 della Costituzione e in particolare ai commi secondo e terzo" (il testo è reperibile in https://www. cortecostituzionale.it/actionSchedaPronuncia.do?param_ecli=ECLI:IT:COST:1973:38).

${ }^{116}$ De Cupis, 1959.

${ }^{117}$ Ivi, pp. 13 ss. e particolarmente alle pp. 17-21 per la significativa digressione in tema di diritto naturale nei suoi rapporti con l'ordinamento giuridico attraverso la lente dei diritti essenziali della personalità, con frequenti riferimenti al pensiero di Del Vecchio.

${ }^{118}$ De Cupis, 1959, pp. 256 ss. e citazioni bibliografiche ivi riportate, in tema di diritto alla riservatezza, sul quale cfr. anche Ligi, 1955; De Cupis, 1954, Carnelutti, 1955, nonché, per i primi accenni a una riflessione dottrinale in tema, Ferrara Santamaria, 1937a; Ravà, 1938, pp. 157 ss.; Ferrara Santamaria, 1939, pp. 923-924. Esemplare, fra le posizioni contrarie alla possibilità di configurare il diritto alla riservatezza in età post-costituzionale è la voce del professore milanese Giovanni Pugliese (Pugliese, 1954), alla quale si contrappone, sempre dalle pagine de I/ Foro Italiano dell'anno 1954, quella di Adriano De Cupis (De Cupis, 1954). Il casus belli è la decisione del Tribunale di Milano, emessa il 14 settembre 1953 per la vicenda che vede protagonista il tenore Caruso, le cui fattezze e la cui vita sono rappresentate, peraltro con scarsa fedeltà al vero, in un'opera cinematografica, senza aver raccolto il preventivo consenso degli eredi dell'artista ormai defunto. La vicenda giudiziaria, salita alla ribalta delle cronache è occasione per puntute schermaglie fra giuristi. La posizione negativa di Pugliese può essere ricondotta alle precedenti tesi negatrici della tutela della riservatezza, esposte in Funaioli, 1954 e Messina, 1947. 
alla non conoscenza altrui dell'immagine del soggetto» ${ }^{119}$. Sul finire degli anni Cinquanta, appare dunque chiaro un ribaltamento di prospettiva, che segue uno sviluppo dogmatico e sistematico interno alla scienza giuridica privatistica: il diritto alla riservatezza pare avere assorbito il diritto sull'immagine, precedendolo da un profilo logico-dogmatico.

$\mathrm{Ma}$ al di fuori di tale rapporto inclusivo, che nel tempo diviene sempre più dominante da un profilo teorico e sistematico, il diritto all'immagine incontrerà nuove possibilità espansive ${ }^{120}$. Ricondotta funzionalmente la sua tutela entro una più complessa concezione di persona, che emerge da una riflessione scientifica trasversale ai campi della sociologia, antropologia, filosofia, biologia, esso volge, come abbiamo poco fa accennato, nella direzione della 'identità personale'.

Sono pochi accenni, questi, che rappresentano solamente l'inizio dei molteplici e incessanti sviluppi e ramificazioni novecentesche di un diritto che, dall'Ottocento a oggi, non cessa di aprire e sollecitare lo sguardo del giurista.

\section{Bibliografia}

Adrian J., Nordemann W., Wandtke A.A. (Hrsg.), 1996: Josef Kohler und der Schutz des geistigen Eigentums in Europa, Berlin

Alpa G., 2006: La cultura delle regole. Storia del diritto civile italiano, Roma-Bari, Laterza

Alpa G., 2018: Diritto civile italiano. Due secoli di storia, Bologna, II Mulino

Alpa G., Resta G., 2019²: I diritti della personalità, in Alpa G., Resta G., Trattato di diritto civile, diretto da Rodolfo Sacco, vol. 3: Le persone e la famiglia, I: Le persone fisiche e i diritti della personalità, Torino, UTET, pp. $145 \mathrm{ss}$.

Alvazzi del Frate P., 2020²: Individuo e comunità. Considerazioni storico-giuridiche sull'individualismo, Torino, Giappichelli

Barberis M., 2000: Filosofia del diritto. Un'introduzione storica, Bologna, il Mulino Bartoli G.(ed.), 2017: I Filosofi del diritto alla 'Sapienza' tra le due Guerre. Atti del Convegno Internazionale, Roma, 21 e 22 ottobre 2014, Roma, Università Sapienza

Bavetta G., 1970: Immagine (diritto alla), in Enciclopedia del diritto, 20, pp. 144156

Bertani M., 2011: Diritto d'autore e connessi, in Ubertazzi L.C. (ed.), La proprietà intellettuale, in Trattato di diritto privato dell'Unione Europea, diretto da Ajani G., Benacchio G.A., vol. XII, Torino, Giappichelli, pp. 222-406

Bobbio N., 2011: Giusnaturalismo e positivismo giuridico (1965). Prefazione di

\footnotetext{
${ }^{119}$ De Cupis, 1959, p. 259.

${ }^{120}$ De Cupis, 1949, pp. 14-15.
} 
Luigi Ferrajoli, Roma-Bari, Laterza

Busnelli F. D.: 1995, Per una rilettura del "diritto delle persone" di cinquant'anni $f a$, in AA.VV., Le ragioni del diritto. Scritti in onore di Luigi Mengoni, t. I: Diritto civile, Milano, Giuffrè, pp. 91-132

Calvo R., 2013: Dusi, Bartolomeo, in I. Birocchi, E. Cortese, A. Mattone, M.N. Miletti (eds.), Dizionario Biografico dei giuristi italiani (XII-XX secolo), 1, Bologna, II Mulino, pp. 789-790

Canestrari S., Ferrando G., Mazzoni C.M., Rodotà S., Zatti P. (eds.), 2011: /I governo del corpo, I, in Trattato di Biodiritto, diretto da S. Rodotà e P. Zatti, Milano, Giuffrè

Cappellini P. 1999: Il fascismo invisibile. Una ipotesi di esperimento storiografico sui rapporti tra codificazione civile e regime, in "Quaderni fiorentini per la storia del pensiero giuridico moderno", 28, pp. 175-292

Caravale M., 2016: Una incerta idea: Stato di diritto e diritti di libertà nel pensiero italiano tra età liberale e fascismo, Bologna, II Mulino

Caroni P., 1996: Saggi sulla storia della codificazione, Milano, Giuffrè

Cassi A.A., 1999: Il bravo funzionario asburgico tra Absolutismus e Aufklärung: il pensiero e l'opera di Karl Anton von Martini (1726-1800), Milano, Giuffrè

Cavanna A., 2005: Storia del diritto moderno in Europa. Le fonti e il pensiero giuridico, 2, Milano, Giuffrè

Cazzetta G., 2011: Codice civile e identità giuridica nazionale. Percorsi e appunti per una storia delle codificazioni moderne, Torino, Giappichelli

Cionti F., 1998: Alle origini del diritto all'immagine. Dall'immagine dipinta, all'immagine fotografata e della cosa, Milano, Giuffrè

Cionti F., 2000: La nascita del diritto sull'immagine, Milano, Giuffrè

De Vita A., 1988: Art. 10, in Commentario del Codice civile Scialoja-Branca, a cura di F. Galgano, Libro I-Delle persone e della famiglia, Delle persone fisiche, art. 1-10, Bologna-Roma, Zanichelli-Edizioni del Foro italiano, pp. 505 ss

Dogliotti M., 1997: Immagine ed identità personale: soggetti forti e soggetti deboli, in "Diritto di famiglia e delle persone", 1, II, pp. 1444-1458

Dogliotti M., 1999²: Le persone fisiche, in Trattato di diritto privato, diretto da P. Rescigno, 2, I, Torino, UTET

Fassò, G., 2006: Storia della filosofia del diritto, vol. III: Ottocento e Novecento, ed. a cura di C. Faralli, Roma-Bari, Laterza

Ferrante R., 2006: Un ruolo per l'interprete: la scienza giuridica italiana tra Code Napoléon e $A B G B$, in "Forum historiae iuris", https://forhistiur.net2006-01ferrante

Fioravanti M., 2021: Lezioni di storia costituzionale. Le libertà fondamentali. Le forme di governo. Le costituzioni del novecento, Torino, Giappichelli 
Furfaro F, 2016: Recezione e traduzione della Pandettistica in Italia tra Otto e Novecento. Le note italiane al Lehrbuch des Pandektenrechts di B. Windscheid, Torino, Giappichelli

Fusar Poli E., 2012: Centro dinamico di forze. I giuristi e l'innovazione scientificotecnologica fra liberismo e autarchia, Milano, Giuffrè

Fusar Poli E., 2018: "Una parte così viva e così importante del diritto»: agli albori dell'insegnamento del Diritto industriale nell'Università italiana, in "Rivista di storia del diritto Italiano", 91, II, pp. 163-214

Fusar Poli E., 2020a: The physical body through the juridical lens: an issue of law of innovation and of innovation in law (XIX-XX cent.), in M. Meccarelli, C. Paixão, C. Roesler (eds.), Innovation and transition in Law: experiences and theoretical settings, Madrid, Dykinson, pp. 247-271

Fusar Poli E., 2020b: Oltraggi d'autore. Questioni novecentesche d'arte, diritto e cripto-censura, in "LawArt. Rivista di Diritto, Arte, Storia", 1, pp. 139-177

Grossi P., 2000: Scienza giuridica italiana. Un profilo storico (1860-1950), Milano, Giuffrè

Grossi P., 2021: II diritto civile in Italia fra moderno e posmoderno. Dal monismo legalistico al pluralismo giuridico, Milano, Giuffrè-Francis Lefebrve

Klippel D., 1982: Historische Wurzeln und Funktionen von Immaterialgüterund Persönlichkeitsrechte im 19. Jahrhundert, in "Zeitschrift für neuere Rechtsgeschichte", pp. 132 ss.

Lacchè L., 2015: Sulla vocazione del giurista italiano. Scienza giuridica, canone eclettico e Italian style tra '800 e '900, in "Rivista italiana per le scienze giuridiche", 6, pp. 233-268

Martello F., 2018: Le implicazioni giuridiche del "progresso tecnico". Categorie, questioni, strumenti nel dibattito tra Otto e Novecento, tesi di dottorato, Università degli Studi di Macerata, ciclo XXXI, rel. M. Meccarelli [https:// u-pad.unimc.it/retrieve/handle/11393/258985/86400/TESI\%20Le\%20 implicazioni\%20giuridiche\%20del\%20progresso\%20tecnico\%20categorie\%20 questioni\%20strumenti\%20nel\%20dibattito\%20tra\%200tto\%20e\%20Novecento.pdf]

Messinetti D., 1983: Personalità (diritti della), in Enciclopedia del diritto, 33, Milano, Giuffrè, pp. 355-406

Mazzarella F., 2007: Nel segno dei tempi. Marchi persone e cose dalla corporazione medievale all'impresa globale, Milano, Giuffrè

Mazzarella F., 2019: Da diritto a paradigma: uso e abuso della proprietà nella disciplina dei diritti d'esclusiva, in "Rivista di diritto civile", 65, IV, pp. 861-889

Meccarelli M., 2011: Diritto giurisprudenziale e autonomia del diritto nelle strategie discorsive della scienza giuridica tra Otto e Novecento, "Quaderni fiorentini per la storia del pensiero giuridico moderno", 40, pp. 721-745 
Montanari B., 2013: Del Vecchio, Giorgio, in I. Birocchi, E. Cortese, A. Mattone, M.N. Miletti (eds.), Dizionario Biografico dei giuristi italiani (XII-XX secolo), 1, Bologna, II Mulino, pp. 744-747

Moscati L., 2001: Sul diritto d'autore tra Codice e leggi speciali, in luris Vincula. Studi in onore di Mario Talamanca, 5, Napoli, Jovene, pp. 497-527

Moscati L., 2015: I diritti morali e la conferenza di Roma del 1928 per la revisione della Convenzione di Berna, in "Quaderni fiorentini per la storia del pensiero giuridico moderno", 44, pp. 465-484

Moscati L., 2020: Diritti d'autore. Storia e comparazione nei sistemi di civil law e di common law, Milano, Giuffrè Francis Lefebvre

Moscati L., 2021: Origins, Evolutions and Comparisons of Moral Rights between Civil and Common Law Systems, "European Business Law Review", 32 (2021), pp. 25-52

Notari S., 2013: Bensa, Paolo Emilio, in I. Birocchi, E. Cortese, A. Mattone, M.N. Miletti (eds.), Dizionario Biografico dei giuristi italiani (XII-XX secolo), 1, Bologna, II Mulino, pp. 218-220

Padoa Schioppa A., 2007: Storia del diritto in Europa. Dal medioevo all'età contemporanea, Bologna, II Mulino

Pino G., 2003a: /l diritto all'identità personale. Interpretazione costituzionale e creatività giurisprudenziale, Bologna, II Mulino

Pino G., 2003b: Teorie e dottrine dei diritti della personalità. Uno studio di metagiurisprudenza analitica, in "Materiali per una storia della cultura giuridica", I, pp. 237-274

Pino G., 2006: /l diritto all'identità personale ieri e oggi. Informazione, mercato, dati personali, in R. Panetta (ed.), Libera circolazione e protezione dei dati personali, I, Milano, Giuffrè, pp. 257-321

Resta G., 2007: Diritti della personalità: problemi e prospettive, in "Il diritto dell'informazione e dell'informatica", 23, pp. 1043-1071

Rondinone N., 2003: Storia inedita della codificazione civile, Milano, Giuffrè

Sciumè A., 2002: I principî generali del diritto nell'ordine giuridico contemporaneo (1837-1942), Torino, Giappichelli

Sciumè A., 2012: Ragione e volontà nella formazione del diritto italiano contemporaneo, in Id. (ed), II diritto come forza. La forza del diritto. Le fonti in azione nel diritto europeo tra medioevo ed età contemporanea, Torino, Giappichelli, pp. 217-262

Solimano S., 2012: Tendenze della civilistica postunitaria, in // contributo italiano alla scienza del pensiero. Ottava appendice: Diritto, Roma, Istituto della enciclopedia Italiana Treccani, pp. 381 ss.

Solimano S., 2013: Fadda, Carlo, in I. Birocchi, E. Cortese, A. Mattone, M.N. Miletti 
(eds.), Dizionario Biografico dei giuristi italiani (XII-XX secolo), 1, Bologna, II Mulino, pp. 813-814

Stolzi I., 2007: L' ordine corporativo. Poteri organizzati e organizzazione del potere nella riflessione giuridica dell'Italia fascista, Giuffrè, Milano

Stolzi I., 2020: I/ secolo nuovo: giuristi e tradizioni nell'Italia del Ventennio, in "Quaderni fiorentini per la storia del pensiero giuridico moderno", 49, pp. 267305

Tartaglia Polcini A., 2013: L'avvento della Costituzione. La prima fase, in A. Tartaglia Polcini, P. Perlingieri (eds.): Novecento giuridico: i civilisti, Napoli, ESI, pp. 215 ss.

Testuzza M.S., 2016: "Ius corporis, quasi ius de corpore disponendi". I/ tractatus de potestate in se ipsum di Baltasar Gómez de Amescua, Milano, Giuffrè

Testuzza M.S., 2019, II fatto della generazione: un ponte gettato a far arco (A proposito di Genesis. XVII/1. La ricerca della paternità. Responsabilità, diritti e affetti, a cura di Stefania Bartoloni e Daniela Lombardi), in "Quaderni Fiorentini per la storia del pensiero giuridico moderno", 48, pp. 785-815

Turelli G., 2013: 'Res incorporales' e 'beni immateriali': categorie affini, ma non congruenti, in A. Sciumè, E. Fusar Poli (eds.), "Afferrare...l'inafferrabile". I giuristi e il diritto della nuova economia industriale fra Otto e Novecento, Giuffrè, Milano, pp 71-88

Viola F., 2016: Introduction: Natural Law Theories in the 20th Century, in A Treatise of Legal Philosophy and General Jurisprudence, vol. 12: Legal Philosophy in the Twentieh Century: The Civil World, t. 2: Main Orientations and Topics, E. Pattaro, C. Roversi (eds.), Springer, Dordrecht, pp 1065-1153

Welser R., 2015: L'ABGB. Un capolavoro di codificazione, in P. Caroni, R. Ferrante (eds.), La codificazione del diritto fra il Danubio e l'Adriatico, Torino, Giappichelli, pp. $15-24$

Zarro M., 2013: Ferrara, Luigi, in I. Birocchi, E. Cortese, A. Mattone, M.N. Miletti (eds.), Dizionario Biografico dei giuristi italiani (XII-XX secolo), 1, Bologna, II Mulino, p. 842

Zeno-Zencovich, 1993: Identità personale, in Digesto delle Discipline PrivatisticheSez. Civile, vol. IX, Torino, UTET, pp. 294-303

Zeno-Zencovich, 1995: Personalità (diritti della), in Digesto delle Discipline Privatistiche-Sez. Civile, vol. XIII, Torino, UTET, pp. 430 ss..

Fonti

Amar M., 1874: Dei diritti degli autori di opere dell'ingegno, Torino, Fratelli Bocca Benjamin W., 1991: Kleine Geschichte der Photographie, 1931, in R. Tiedemann, 
H. Schweppenhäuser (Hrsg.), Gesammelte Schriften, Bd.2.: Aufsätze, Essays, Vorträge, I, Frankfurt am Main, Suhrkamp, pp. 368-385

Bigeon A.-A., 1894 : La photographie et le droit. Nouvelle édition revue et augmentée, Paris, C. Mendels

Bucciante G., 1911: La fotografia ed il diritto sulla propria immagine, Ancona

Campogrande V., 1904: // «jus in se ipsum» in rapporto alla natura del diritto sulla propria immagine, in "La Legge", pp. 811-828, pp. 915-924

Carnelutti F., 1955: Diritto alla vita privata, in "Rivista trimestrale di diritto pubblico", I, pp. 3 ss.

Coviello N., 1924: Manuale di diritto civile italiano. Parte generale, Milano, Società Editrice Libraria

Chironi G.P., Abello L., 1904: Trattato di diritto civile italiano, vol. I: Parte generale, Torino, Fratellio Bocca

De Cupis A., 1949: Il diritto all'identità personale, vol. I: Il diritto al nome, Milano, Giuffrè

De Cupis A., 1951, Ancora in tema di offesa morale per mezzo della divulgazione cinematografica, in "II Foro Italiano", 75, I, cc. 149-156

De Cupis A., 1954: I/ diritto alla riservatezza esiste, in "Il Foro Italiano", 77, cc. 89-98

De Cupis A., 1956: La persona umana nel diritto privato, in "Il Foro Italiano", 79, cc. 77-86

De Cupis A., 1959: I diritti della personalità, in Trattato di diritto civile e commerciale, diretto da A. Cicu, F. Messineo, vol. 4, t. I: Teoria generale, diritto alla vita e all'integrità fisica, diritto sulle parti staccate del corpo e sul cadavere, diritto alla libertà, diritto all'onore e alla riservatezza, Milano, Giuffrè, pp. 286 ss.

De Sanctis V., 1937: II diritto sulla propria immagine nel nuovo codice civile, in "II diritto di autore", 7, pp. 7 ss.

Degni F., 1939: Le persone fisiche e di diritti della personalità, in Trattato di diritto civile italiano, diretto da F. Vassalli, 2, I, Torino, UTET

Del Vecchio G., 1921: Sui principi generali del diritto, Modena (anche in "Rivista Italiana per le Scienze Giuridiche", 8 (2017), pp. 15-63)

Del Vecchio G., 1923: Diritto e personalità, Bologna, Zanichelli

Dusi B, 1906: Cenni intorno al diritto alla immagine, in "Studi senesi" vol. 23: Scritti giuridici e di scienze economiche pubblicati in onore di Luigi Moriani nel XXXV anno del suo insegnamento, 2, pp. 209 ss.

Dusi B., 1907: Del diritto all'immagine, in "Rivista di diritto commerciale", II, pp. 431

Fadda C., Bensa P.E., 1926: Diritto delle Pandette di Bernardo Windscheid, vol. IV: Note ai Libri I. - Del diritto in genere, II. - Dei diritti in generale, Torino, UTET 
Falco G., 1938: Identità personale, in Nuovo digesto italiano, 6, Torino, UTET, p. 649

Ferrara L., 1901: Caso tipico in materia di proprietà fotografica, in "Il Filangieri", pp. 481-502

Ferrara L., 1903: Intorno al diritto sulla propria immagine, in "Giurisprudenza italiana", 55, IV, pp. 279 ss.

Ferrara L., 1937a: Diritti d'autore e diritti della fede, in "Monitore dei tribunali", pp. 290 ss.

Ferrara L., 1937b: "Jus imaginis" e centenario della fotografia, in "Il diritto d'autore", pp. 443-464

Ferrara L., 1938: Riproduzione abusiva di ritratto altrui a scopo di "réclame", in "Diritto d'autore", pp. 501-514

Ferrara L., 1942: Il diritto sulla propria immagine nel nuovo codice civile e nella nuova legge sul diritto d'autore, Roma, Edizioni Italiane

Ferrara Santamaria M., 1937a: Il diritto alla illesa intimità privata, in "Rivista di diritto privato", pp. 168 ss.

Ferrara Santamaria M. , 1937b: Il rapporto di "inerenza" dei diritti alla persona, In "Rivista di diritto civile", I, pp. 338 ss.

Ferrara Santamaria M., 1939: Persona (diritti della), in Nuovo Digesto Italiano, 9, pp. 912-926

Funaioli C.A., 1954: Diritto cinematografico e tutela della personalità, in "Giustizia civile", pp. 581 ss.

Keyssner H., 1896: Das Recht am eigenen Bilde, Berlin, Guttentag

Kohler J., 1903: Das Eigenbild im Recht, Berlin, Guttentag

Kohler J., 1909: Lehrbuch der Rechtsphilosophie, Berlin, Rothshild

Ligi, F., 1954: Alcune questioni circa il diritto all'immagine, in "Il Foro Italiano", 77, cc. $1185-1192$

Ligi F., 1955: /l diritto alle vicende e la sfera della personalità, in "II Foro Italiano", 78, cc. 385-404

Maroi F., 1940: Delle persone fisiche, in Commentario del Codice civile diretto da M. D’Amelio, E. Finzi, Libro primo. Delle persone e della famiglia, 1, Firenze, Barbera, pp. 81-104

Messina S., 1947: Le indiscrezioni artistiche e letterarie, in "Il diritto di autore", pp 287 ss.

Musatti A., 1954: Appunto sul diritto alla riservatezza, in "Il Foro Italiano", 77, cc. 183-188

Piola Caselli E., 1904: Del diritto di autore sui ritratti e busti in rapporto al cosiddetto "diritto sull'immagine propria", in "II Foro Italiano", I, pp. 633 ss. 
Piola Caselli E., 1927²: Trattato del diritto di autore e del contratto di edizione, Napoli-Torino, UTET

Piola Caselli E., 1943: Codice del diritto d'autore: commentario della nuova Legge 22 aprile 1941-XIX n. 633 corredato dei lavori preparatori e di un indice analitico delle leggi interessanti la materia, Torino, UTET

Pouillet E., 1879: Traité théorique et pratique de la propriété littéraire et artistique et du droit de représentation, Paris, Marchal, Billard \& C.

Pugliese G, 1954: Il preteso diritto alla riservatezza e le indiscrezioni cinematografiche, in "Il Foro Italiano", 77, I, cc. 115-134

Ravà A., 1901: I diritti sulla propria persona nella scienza e nella filosofia del diritto, Torino, Fratelli Bocca

Ravà A., 1938: Istituzioni di diritto privato, Padova, Padova, CEDAM

Ricca Barberis M., 1903: /l diritto alla propria figura, in "Rivista del diritto commerciale", I, pp. 192 ss.

Ricca Barberis M., 1903: Il consenso alla divulgazione del ritratto e la sua revocabilità, in "Rivista del diritto commerciale", II, pp. 440 ss.

Ricca Barberis M., 1905: Sulla capacità a disporre dell'immagine, in "Rivista di diritto commerciale", l, pp. 441 ss.

Ricca Barberis M., 1958: /l diritto all'immagine, in "Rivista di diritto civile", IV, 2, pp. 226-230

Rosmini E., 1890: Legislazione e giurisprudenza sui diritti d'autore. Trattato dei rapporti fra autori e editori, impresarii, direttori teatrali e col pubblico..., Milano, Hoepli

Valerio E., 1926: La nuova legge sul diritto di autore e relativo regolamento. Con cenni sulla evoluzione storico-giuridica sul diritto di autore sino all'era fascista, Milano, Morreale

Valerio E., 1930: Disposizioni sul diritto sul diritto d'autore. Conferenza internazionale di Roma sul diritto di autore 7 maggio - 2 giugno 1928, Roma, Libreria del littorio

Vercellone P., 1959: /l Diritto sul proprio ritratto, Torino, UTET

Visco A., 1926: Il diritto sulla propria immagine seconda la nuova Legge sui diritti d'autore, Tivoli, Tip. Meschini

Von Blume, 1903: Il diritto di proprietà sulla propria immagine (trad. it.), In "La legge", pp. 723 ss.. 
